\title{
ONE-DIMENSIONAL DISTRIBUTIONS OF SUBORDINATORS WITH UPPER TRUNCATED LÉVY MEASURE, AND APPLICATIONS
}

\author{
SHAI COVO, ${ }^{*}$ Bar Ilan University
}

\begin{abstract}
Given a pure-jump subordinator (i.e. nondecreasing Lévy process with no drift) with continuous Lévy measure $v$, we derive a formula for the distribution function $F_{S}(x ; t)$ at time $t$ of the associated subordinator whose Lévy measure is the restriction of $v$ to $(0, s]$. It will be expressed in terms of $v$ and the marginal distribution function $F(\cdot ; t)$ of the original process. A generalization concerning an arbitrary truncation of $v$ will follow. Under certain conditions, an analogous formula will be obtained for the $n$th derivative, $\partial^{n} F_{s}(x ; t) / \partial x^{n}$. The requirement that $v$ is continuous is shown to have no intrinsic meaning. A number of interesting results involving the size ordered jumps of subordinators will be derived. An appropriate approximation for the small jumps of a gamma process will be considered, leading to a revisiting of the generalized Dickman distribution.
\end{abstract}

Keywords: Subordinator; Lévy process; compound Poisson process; truncated Lévy measure; ordered jumps; gamma process; small jumps; weak convergence; Dickman distribution

2000 Mathematics Subject Classification: Primary 60G51

Secondary 60E07; 60F17

\section{Introduction}

In this section we provide some basic notation and elementary facts to be used in this paper. For a complete account on the theory of Lévy processes, we refer the reader to the comprehensive monograph of Sato [17].

Let $X=\{X(t): t \geq 0\}$ be a pure-jump subordinator with continuous Lévy measure $v$. The trivial case $X \equiv 0$ will be implicitly excluded in the sequel. Furthermore, let $X_{S}=\left\{X_{S}(t)\right.$ : $t \geq 0\}$ be the pure-jump subordinator whose Lévy measure is the restriction of $v$ to $(0, s]$, where $s$ is an arbitrary positive constant (the case $X_{s} \equiv 0$ is not excluded), and let $F(x ; t)$ and $F_{S}(x ; t)$ denote the distribution functions (DFs) at time $t>0$ of $X$ and $X_{s}$, respectively. Throughout this paper, the two processes may or may not be independent, depending on the context.

We recall [17] that a Lévy process with infinite Lévy measure has a continuous distribution for any $t>0$ and that a compound Poisson process (CPP) has a continuous distribution on $\mathbb{R} \backslash\{0\}$, for $t>0$, if and only if its Lévy measure is continuous.

It is a key property of Lévy processes in general that their jumps form a Poisson random measure on $(0, \infty) \times(\mathbb{R} \backslash\{0\})$ with intensity $\mathrm{d} t \times \pi(\mathrm{d} x), \pi$ being the (arbitrary) Lévy measure

Received 21 November 2007; revision received 18 December 2008.

* Postal address: Department of Mathematics, Bar Ilan University, 52900 Ramat-Gan, Israel.

Email address: green355@netvision.net.il

This paper is part of the author's PhD thesis, prepared at Bar Ilan University under the supervision of Professor E. Merzbach. This work was supported by the Doctoral Fellowship of Excellence, Bar Ilan University. 
(see, e.g. [17, Chapter 4]). Thus, in particular, the number of jumps before time $t$ which take values in some Borel set $B$ is Poisson distributed with mean $t \pi(B)$, where, by convention, Poisson $(0)=0$ and Poisson $(\infty)=\infty$. In the subordinator case, $\pi$ is concentrated on $(0, \infty)$ and $\int(x \wedge 1) \pi(\mathrm{d} x)<\infty$; by the well-known Lévy-Khintchine formula for subordinators, the Laplace transform at time $t$ of the corresponding pure-jump subordinator, say $Y$, is given by

$$
\mathrm{E}\left[\mathrm{e}^{-\omega Y(t)}\right]=\exp \left[t \int_{(0, \infty)}\left(\mathrm{e}^{-\omega x}-1\right) \pi(\mathrm{d} x)\right], \quad \omega \geq 0 .
$$

The size ordered jumps of subordinators will play an important role in this paper.

Let $V_{i}(t)$ denote the $i$ th largest jump of $X$ before time $t$. If $v$ is infinite then $X$ has a countably infinite number of jumps in finite intervals and, hence, $V_{i}(t)>0$ for all $i$. If $v$ is finite then $X$ is a CPP and, hence, $V_{i}(t)=0$ for all $i>m$, where $m$ is the number of jumps before time $t$ (possibly 0 ). In any case, by virtue of $v$ being continuous, $V_{i}(t)$ will (almost surely) be strictly greater than $V_{i+1}(t)$, provided that the first is not 0 .

From now on, it will be convenient to denote by $\Lambda$ the tail of $v$ :

$$
\Lambda(u)=v((u, \infty)), \quad u \geq 0 .
$$

The distribution of $V_{n}(t), n \geq 1$, is given by (cf. [3, Equation (20)])

$$
P_{V_{n}(t)}(\mathrm{d} u)=\frac{t^{n} \Lambda(u)^{n-1}}{(n-1) !} \mathrm{e}^{-t \Lambda(u)} v(\mathrm{~d} u), \quad u>0,
$$

with the following positive mass at 0 for the CPP case:

$$
\mathrm{P}\left(V_{n}(t)=0\right)=\sum_{i=0}^{n-1} P_{i}(t \Lambda(0))
$$

where

$$
P_{k}(\lambda)=\frac{\lambda^{k}}{k !} \mathrm{e}^{-\lambda}, \quad k=0,1,2, \ldots,
$$

are the Poisson probabilities. Intuitively, (1.2) comes from $P_{n-1}(t \Lambda(u)) P_{1}(t v(\mathrm{~d} u))$.

Whether $v$ is finite or infinite, standard properties of Poisson random measures, combined with the continuity of $v$, imply that, conditioned on $V_{n}(t)=u, u>0$, the random variable (RV) $\sum_{i=n+1}^{\infty} V_{i}(t)$ is distributed according to the law at time $t$ of a pure-jump subordinator whose Lévy measure is $v$ restricted to $(0, u]$.

The rest of the paper is organized as follows. In Section 2 we formulate and prove (in two different ways) the fundamental result of this paper, Theorem 2.1 , which expresses $F_{S}(x ; t)$ in terms of $F(\cdot ; t)$ and $v$. A generalization concerning an arbitrary truncation of $v$ is given in Theorem 2.2. Next, in Corollary 2.1, we give the analog of Theorem 2.1 for the density function $\partial F_{S}(x ; t) / \partial x$, provided that $F(\cdot ; t)$ is absolutely continuous (on $\left.\mathbb{R}\right)$. We further develop this analog in Proposition 2.1, stating a continuous variant for the derivatives $\partial^{j} F_{S}(x ; t) / \partial x^{j}$ under suitable regularity conditions on $F(\cdot ; t)$ and $v$. A sketch of the proof is given in Appendix A. A generalization of Theorem 2.1 and Corollary 2.1 to the case where the Lévy measure is not assumed to be continuous, noticed by the author after the paper was ready for acceptance, is provided in Appendix B. In Section 3 we derive a series of distributional results involving the ordered jumps of subordinators, a basic one being a formula for the tail DF of the ratio 
$V_{n}(t) /\left(\sum_{i=n}^{\infty} V_{i}(t)+b t\right), b \geq 0$ (Proposition 3.1), which, together with its Corollary 3.1, significantly extends a previous result by Perman [14]. In Section 4 we consider an appropriate approximation for the small jumps of a gamma process $X$, based on weak convergence of the scaled subordinator $X_{\varepsilon} / \varepsilon$ as $\varepsilon \rightarrow 0$ to a subordinator whose one-dimensional marginals are generalized Dickman distributions (see Section 4.1 for the definition and Proposition 4.1 for the proof). Using Theorem 2.1, we derive the DF of $X_{\varepsilon}(t) / \varepsilon$ (see (4.6)). We then obtain the DF of the generalized Dickman law, denoted by $\operatorname{GD}(\theta)$, as well as its first $\lceil\theta\rceil$ derivatives (Proposition 4.2), and compare with several existing results in the literature.

\section{Main results}

\subsection{The distribution function}

With the above notation, define, for $k=1,2, \ldots$ and $x>k s$ (or $x \in \mathbb{R}$ in general),

$$
A_{s ; k}(x ; t)=\int_{C_{s ; k}(x)} F\left(x-\sum_{i=1}^{k} u_{i} ; t\right) v\left(\mathrm{~d} u_{1}\right) \cdots v\left(\mathrm{~d} u_{k}\right),
$$

where

$$
C_{s ; k}(x)=\left\{\boldsymbol{u} \in \mathbb{R}^{k}: s<u_{1}<\cdots<u_{k}, u_{1}+\cdots+u_{k} \leq x\right\} .
$$

The multiple integral can be calculated as an iterated integral, with the integration variables ranging as follows:

$$
s<u_{1}<\frac{x}{k}, \quad u_{1}<u_{2}<\frac{x-u_{1}}{k-1}, \quad \ldots, \quad u_{k-1}<u_{k}<x-\sum_{i=1}^{k-1} u_{i} .
$$

It will prove useful later on to define $\int_{C_{s ; k}(x)} \cdot=$. if $k=0$ (say for $x>0$ ). Thus, e.g. $A_{s ; 0}(x ; t)=F(x ; t)$.

Theorem 2.1. The distribution function of $X_{s}(t)$ is given by

$$
F_{S}(x ; t)=\mathrm{e}^{t \Lambda(s)}\left(F(x ; t)+\sum_{k=1}^{\lceil x / s\rceil-1}(-t)^{k} A_{s ; k}(x ; t)\right), \quad x \in \mathbb{R} .
$$

It is sometimes preferable, as, e.g. in the proofs of Theorem 2.1, below, to put the exponential factor on the left-hand side.

Proof of Theorem 2.1. Clearly, we may assume that $x \geq 0$. The proof is then by induction on $\lceil x / s\rceil$. The cases $\lceil x / s\rceil=0,1$ (i.e. $0 \leq x \leq s$ ) follow easily. Indeed, conditionally on the event $\mathcal{A}$ that $X$ has no jump of size larger than $s$ before time $t$ (whose probability is $P_{0}(t \Lambda(s))$ ), the $\mathrm{RV} X(t)$ is distributed as $X_{s}(t)$, and, thus, $\mathrm{P}\left(X_{s}(t) \leq x\right)=\mathrm{P}(X(t) \leq x \mid \mathcal{A})=\mathrm{P}(X(t) \leq$ $x) / \mathrm{e}^{-t \Lambda(s)}$.

Now suppose that the statement holds for $\lceil x / s\rceil=0,1, \ldots, m, m \in \mathbb{N}$, and set $\lceil x / s\rceil=$ $m+1$. Note that in particular $x>s$. By the law of total probability, conditioning on $V_{1}(t)$ (the largest jump of $X$ before $t$ ), and using (1.2), (1.3), and the argument that followed (all of them for $n=1$ ), we obtain

$$
\mathrm{P}(X(t) \leq x)=\exp [-t \Lambda(0)]+\int_{0}^{x} \mathrm{P}\left(X_{u_{1}}(t) \leq x-u_{1}\right) \exp \left[-t \Lambda\left(u_{1}\right)\right] t v\left(\mathrm{~d} u_{1}\right),
$$


where $X_{u_{1}}$ is a pure-jump subordinator whose Lévy measure is $v$ restricted to $\left(0, u_{1}\right]$, and, interpreting $\mathrm{e}^{-\infty}$ as 0 in the infinite measure case, $\Lambda(0)=\infty$. Similarly, conditioning on the largest jump of $X_{s}$ before $t$, and letting $\Lambda_{s}$ denote the truncated tail of $v$ defined by $\Lambda_{s}(u)=$ $v((u, s])$, we obtain

$$
\mathrm{P}\left(X_{S}(t) \leq x\right)=\exp \left[-t \Lambda_{s}(0)\right]+\int_{0}^{s} \mathrm{P}\left(X_{u_{1}}(t) \leq x-u_{1}\right) \exp \left[-t \Lambda_{s}\left(u_{1}\right)\right] t v\left(\mathrm{~d} u_{1}\right) .
$$

From (2.5) and (2.6), it follows straightforwardly that

$$
F_{S}(x ; t) \mathrm{e}^{-t \Lambda(s)}=F(x ; t)-t \int_{s}^{x} F_{u_{1}}\left(x-u_{1} ; t\right) \exp \left[-t \Lambda\left(u_{1}\right)\right] v\left(\mathrm{~d} u_{1}\right),
$$

with $F_{u_{1}}(\cdot ; t)$ being the DF of $X_{u_{1}}(t)$. Now consider the partition of $[s, x)$ into the intervals

$$
I_{k}= \begin{cases}{\left[\frac{x}{k+1}, \frac{x}{k}\right),} & k=1, \ldots, m-1, \\ {\left[s, \frac{x}{m}\right),} & k=m .\end{cases}
$$

It is easy to check that $u_{1} \in I_{k}$ implies that $\left\lceil\left(x-u_{1}\right) / u_{1}\right\rceil=k$, so we apply the induction hypothesis to $F_{u_{1}}\left(x-u_{1} ; t\right) \exp \left[-t \Lambda\left(u_{1}\right)\right]$ for each interval $I_{k}$ separately, where $u_{1}$ and $x-u_{1}$ play the role of $s$ (which is arbitrary) and $x$, respectively. Each $I_{k}$ then accounts for $k$ integrand terms. Recalling (2.3), the proof is completed by collecting the identical terms and joining the corresponding intervals.

Remark 2.1. We observe that the integral defining $A_{s ; k}(x ; t)$ is invariant with respect to the ordering of the $u_{i} \mathrm{~s}$ in (2.2). Thus, $A_{s ; k}(x ; t)$ can be equivalently written as

$$
A_{s ; k}(x ; t)=\frac{1}{k !} \int_{D_{s ; k}(x)} F\left(x-\sum_{i=1}^{k} u_{i} ; t\right) v\left(\mathrm{~d} u_{1}\right) \cdots v\left(\mathrm{~d} u_{k}\right),
$$

where

$$
D_{s ; k}(x)=\left\{\boldsymbol{u} \in \mathbb{R}^{k}: u_{1}>s, \ldots, u_{k}>s, u_{1}+\cdots+u_{k} \leq x\right\} .
$$

The multiple integral can be calculated as an iterated integral, with the integration variables ranging as follows:

$$
s<u_{1}<x-(k-1) s, \quad s<u_{2}<x-(k-2) s-u_{1}, \quad \ldots, \quad s<u_{k}<x-\sum_{i=1}^{k-1} u_{i} .
$$

From this follows the recursive convolution relation (cf. (2.9), below)

$$
A_{s ; k+1}(x ; t)=\frac{1}{k+1} \int_{s}^{x-k s} A_{s ; k}(x-u ; t) v(\mathrm{~d} u), \quad x>(k+1) s,
$$

which is, recalling the convention $A_{s ; 0}(x ; t)=F(x ; t)$, valid for $k=0$ as well. We note that upper bounds for $A_{s ; k}(x ; t)$ may be derived based on $(2.7)$, a naive one being

$$
A_{s ; k}(x ; t) \leq \frac{1}{k !} F(x-k s ; t)[v((s, x-(k-1) s))]^{k}, \quad x>k s .
$$


In retrospect, it would have been more appropriate to define $A_{s ; k}(x ; t)$ as in (2.7), but without the leading factor of $1 / k$ !. We are now going to see why.

The referee pointed out the following fundamental observation. It follows readily from (2.7) that the $A_{s ; k}(x ; t)$ integrals are obtained by convolution of the DF $F(\cdot ; t)$ with convolution powers of the finite Lévy measure

$$
\lambda_{s}(\mathrm{~d} y)=\mathbf{1}_{(s, \infty)}(y) v(\mathrm{~d} y) .
$$

Namely, we have, for $k=0,1,2, \ldots$ and $x>0$,

$$
A_{s ; k}(x ; t)=\frac{1}{k !}\left(F * \lambda_{s}^{* k}\right)(x ; t) .
$$

(When $k=0, \lambda_{s}^{* k}$ is understood to be the Dirac measure at 0 .)

More generally, we note for later use that it holds that

$$
\int_{(k s, x]} f(x-u) \lambda_{s}^{* k}(\mathrm{~d} u)=\int_{D_{s ; k}(x)} f\left(x-\sum_{i=1}^{k} u_{i}\right) \pi\left(\mathrm{d} u_{1}\right) \cdots \pi\left(\mathrm{d} u_{k}\right), \quad k \geq 1,
$$

for any nonnegative measurable function $f$ and subordinator measure $\pi\left(\lambda_{s}=\pi_{\mid(s, \infty)}\right)$.

We stress that the convolution version, though not so elegant in explicit form, is more convenient for analysis. For concrete examples, see the proof of Corollary 2.1, below, and the first paragraph of Appendix A. A computational advantage is that the $\lambda_{s}^{* k}(\mathrm{~d} y)$ are serviceable for calculating the integrals of (2.10) for different functions $f$ (consider the $A_{s ; k}^{(j)}(x ; t)$ defined in (2.16), below). A nice application of (2.9) to a CPP with exponential jumps is provided in Example 2.1, below.

As indicated in the introduction, Theorem 2.1 and Corollary 2.1 are generalized in Appendix B to the case where the Lévy measure is not assumed to be continuous. The author would not have discovered this extension without the referee's observation (2.9).

The referee also pointed out the following illuminating remark.

Remark 2.2. There is an (easy) dual formula to (2.4):

$$
F(x ; t)=\mathrm{e}^{-t \Lambda(s)}\left(F_{S}(x ; t)+\sum_{k=1}^{\lceil x / s\rceil-1} t^{k} B_{s ; k}(x ; t)\right), \quad x \in \mathbb{R},
$$

with

$$
B_{s ; k}(x ; t)=\frac{1}{k !}\left(F_{s} * \lambda_{s}^{* k}\right)(x ; t)
$$

or, alternatively,

$$
B_{s ; k}(x ; t)=\frac{1}{k !} \int_{D_{s ; k}(x)} F_{s}\left(x-\sum_{i=1}^{k} u_{i} ; t\right) v\left(\mathrm{~d} u_{1}\right) \cdots v\left(\mathrm{~d} u_{k}\right) .
$$

This corresponds to the decomposition $X(t)=X_{s}(t)+X^{s}(t)$, where $X^{s}$ is a CPP with Lévy measure $\lambda_{s}$, independent of $X_{s}$.

Remark 2.3. In fact, a simple generalization of the relation

$$
F_{s}(x ; t) \mathrm{e}^{-t \Lambda(s)}=F(x ; t), \quad x \leq s
$$

(given in (3.10), below), will provide the basis for a whole subsection (Section 3.2). 
We will now give an alternative proof of Theorem 2.1 that offers an intuitive explanation of (2.4), which is not apparent from the inductive approach. Moreover, and much more importantly, it implies a generalization of this theorem, as given in Theorem 2.2, below. (See also Remark 2.4 within this context.)

An alternative proof of Theorem 2.1. We begin our alternative proof by writing

$$
\mathrm{P}(X(t) \leq x)=\mathrm{P}(X(t) \leq x, \mathcal{A})+\mathrm{P}\left(X(t) \leq x, \mathcal{A}^{\mathrm{c}}\right),
$$

where, as before, $\mathcal{A}$ is the event that $X$ has no jump of size larger than $s$ before $t$. The case $0 \leq x \leq s$ is straightforward, so we assume that $x>s$. Since $\mathrm{P}(X(t) \leq x, \mathcal{A})=$ $\mathrm{e}^{-t \Lambda(s)} \mathrm{P}\left(X_{S}(t) \leq x\right)$, it suffices to show that

$$
\mathrm{P}(X(t) \leq x, \mathcal{B})=\sum_{k=1}^{\lceil x / s\rceil-1}(-1)^{k+1} t^{k} A_{s ; k}(x ; t),
$$

where $\mathscr{B}$ is the event that $X$ has at least one jump of size in $(s, x]$ before $t$.

Let us now partition $(s, x]$ into the $N$ subintervals ( $N$ is a large integer)

$$
\left(u, u+\frac{x-s}{N}\right], \quad u \in T ; \quad T=\left\{s+j \frac{x-s}{N}: j=0,1, \ldots, N-1\right\},
$$

and let $\mathcal{C}_{u}$ denote the event that $X$ has a jump of size in $(u, u+(x-s) / N]$ before $t$. Then $\mathscr{B}=\bigcup_{u \in T} \mathcal{C}_{u}$, and, hence,

$$
\mathrm{P}(X(t) \leq x, \mathcal{B})=\mathrm{P}\left(\bigcup_{u \in T}\left[X(t) \leq x, \mathcal{C}_{u}\right]\right) .
$$

We next apply the inclusion-exclusion principle to the right-hand side probability, noting that the total number of jumps before $t$ with values in $(s, x]$ is at most $\lceil x / s\rceil-1$ and their sum does not exceed $x$. This leads to

$$
\mathrm{P}(X(t) \leq x, \mathscr{B})=\sum_{k=1}^{\lceil x / s\rceil-1}(-1)^{k+1} \sum_{\begin{array}{r}
u_{1}, \ldots, u_{k} \in T \\
u_{1}<\cdots<u_{k} \\
u_{1}+\cdots+u_{k}<x
\end{array}} \mathrm{P}\left(X(t) \leq x, \mathcal{C}_{u_{1}}, \ldots, \mathcal{C}_{u_{k}}\right) .
$$

Letting $N \rightarrow \infty$ and using standard properties of Poisson random measures, the inner sum in (2.13) is replaced by the integral

$$
\int_{C_{s ; k}(x)} \mathrm{P}\left(X(t) \leq x-\sum_{i=1}^{k} u_{i}\right) t^{k} v\left(\mathrm{~d} u_{1}\right) \cdots v\left(\mathrm{~d} u_{k}\right) .
$$

Thus, we arrive at (2.12), and so we are done.

From this proof we can easily deduce a generalization of Theorem 2.1 concerning an arbitrary truncation of $v$. Suppose that $B$ is a Borel set with infimum $s>0$, and consider the pure-jump subordinator $X \backslash B$ whose law is that of the process obtained from $X$ by removing all jumps of size in $B$ from $X$ (that is, its Lévy measure is the restriction of $v$ to $(0, \infty) \backslash B)$. Furthermore, let $F_{\backslash B}(\cdot ; t)$ denote the DF at time $t>0$ of this process. Then, considering the event $\mathcal{A}^{\prime}$ that $X$ has no jump of size in $B$ before $t$ in the proof above (instead of $\mathcal{A}$ ), the following result can be established. 
Theorem 2.2. The distribution function of $X_{\backslash B}(t)$ is given by

$$
\begin{aligned}
& F_{\backslash B}(x ; t) \\
& =\mathrm{e}^{t v(B)}\left(F(x ; t)+\sum_{k=1}^{\lceil x / s\rceil-1}(-t)^{k}\right. \\
& \left.\times \int_{C_{s ; k}(x) \cap B^{k}} F\left(x-\sum_{i=1}^{k} u_{i} ; t\right) v\left(\mathrm{~d} u_{1}\right) \cdots v\left(\mathrm{~d} u_{k}\right)\right), \quad x \in \mathbb{R} .
\end{aligned}
$$

However, all of the applications presented in this paper (Sections 3 and 4) correspond to the special case $B=(s, \infty)$.

Remark 2.4. Here we would like to draw attention to the following result, which is immediate from the analog of (2.12) for the case of arbitrary truncation:

$$
\begin{aligned}
\mathrm{P}(X(t) & \leq x \mid \tilde{\mathcal{B}}) \\
= & \frac{1}{1-\mathrm{e}^{-t v(B)}} \\
& \times \sum_{k=1}^{\lceil x / s\rceil-1}(-1)^{k+1} t^{k} \int_{C_{s ; k}(x) \cap B^{k}} F\left(x-\sum_{i=1}^{k} u_{i} ; t\right) v\left(\mathrm{~d} u_{1}\right) \cdots v\left(\mathrm{~d} u_{k}\right), \quad x \in \mathbb{R},
\end{aligned}
$$

where $\tilde{B}$ is the event that $X$ has at least one jump of size in $B$ before $t$, assuming that $v(B)>0$. This result, which is not pertinent to our present topic, deserves a good deal of consideration.

We conclude this subsection with the following elegant result.

Example 2.1. Let $X$ be a CPP with arrival rate $\lambda>0$ and exponential $(\theta)$ jumps. That is, $X$ is a pure-jump subordinator with Lévy measure given by $v(\mathrm{~d} x)=\lambda \theta \mathrm{e}^{-\theta x} \mathrm{~d} x, x>0$. Furthermore, let $\gamma(a, b)$ denote the lower incomplete gamma function, i.e. $\gamma(a, b)=\int_{0}^{b} z^{a-1} \mathrm{e}^{-z} \mathrm{~d} z$. From the well-known identity

$$
\frac{\gamma(j, x)}{(j-1) !}=\left(1-\mathrm{e}^{-x} \sum_{i=0}^{j-1} \frac{x^{i}}{i !}\right), \quad j=1,2, \ldots
$$

(expressing the DF of the Erlang distribution with shape parameter $j$ and rate/scale parameter 1), it follows, upon conditioning on the number of jumps, that the DF of $X(t)$ can be written as

$$
F(x ; t)=1-\mathrm{e}^{-\theta x} \sum_{j=0}^{\infty} \frac{\mathrm{e}^{-\lambda t}(\lambda t)^{j}}{j !} \sum_{i=0}^{j-1} \frac{(\theta x)^{i}}{i !}, \quad x>0
$$

$\left(F(0 ; t)=\mathrm{e}^{-\lambda t}\right)$. In our case, the measure $\lambda_{s}^{* k}, k \geq 1$, in (2.9) admits a particularly simple expression:

$$
\lambda_{s}^{* k}(\mathrm{~d} y)=\frac{(\lambda \theta)^{k}}{(k-1) !}(y-k s)^{k-1} \mathrm{e}^{-\theta y} \mathrm{~d} y, \quad y>k s,
$$


which follows immediately by induction. By calculating the (cumbersome but elementary) integral $\int_{k s}^{x} F(x-u ; t) \lambda_{s}^{* k}(\mathrm{~d} u)$, i.e. $k ! A_{s ; k}(x ; t)$, we have found that

$$
A_{s ; k}(x ; t)=\frac{\lambda^{k}}{k !}\left(\mathrm{e}^{-\theta k s}-\mathrm{e}^{-\theta x} \sum_{j=0}^{\infty} \frac{\mathrm{e}^{-\lambda t}(\lambda t)^{j}}{j !} \sum_{i=0}^{k+j-1} \frac{(\theta(x-k s))^{i}}{i !}\right), \quad x>k s .
$$

(Note that it holds for $k=0$ as well.)

\subsection{The derivatives}

In this subsection we shall give the analog of Theorem 2.1 for the derivatives $\partial^{j} F_{S}(x ; t) / \partial x^{j}$ in the infinite measure case. We first introduce the following notation.

Henceforth, we let $F^{(j)}(\cdot ; t)$ and $F_{s}^{(j)}(\cdot ; t)$ respectively stand as abbreviations for the functions $\partial^{j} F(\cdot ; t) / \partial x^{j}$ and $\partial^{j} F_{S}(\cdot ; t) / \partial x^{j}$ (possibly in an almost everywhere sense if $j=1$ ). The analog of $A_{s ; k}(x ; t)$ in $(2.1)$ is denoted by $A_{s ; k}^{(j)}(x ; t)$ and defined by

$$
A_{s ; k}^{(j)}(x ; t)=\int_{C_{s ; k}(x)} F^{(j)}\left(x-\sum_{i=1}^{k} u_{i} ; t\right) v\left(\mathrm{~d} u_{1}\right) \cdots v\left(\mathrm{~d} u_{k}\right) .
$$

In what follows, the right-hand side of (2.16) will be a well-defined Lebesgue integral-and, hence, Remark 2.1 and in turn (2.10) can be applied-except in one case where a different interpretation is required (see the sentence after (2.20), below).

Before we give the following corollary of Theorem 2.1, we note the simple fact that $X(t)$ is absolutely continuous if and only if $X_{S}(t)$ is ( $v$ is then, of course, infinite). For a proof, consider the decomposition of $X(t)$ given at the end of Remark 2.2. We also observe that (2.18), below, implies the almost everywhere finiteness of $A_{s ; k}^{(1)}(x ; t)$.

Corollary 2.1. Suppose that $F(\cdot ; t)$, and, hence, $F_{S}(\cdot ; t)$, is absolutely continuous (on $\left.\mathbb{R}\right)$. Then the density function $F_{s}^{(1)}(\cdot ; t)$ is given by

$$
F_{s}^{(1)}(x ; t)=\mathrm{e}^{t \Lambda(s)}\left(F^{(1)}(x ; t)+\sum_{k=1}^{\lceil x / s\rceil-1}(-t)^{k} A_{s ; k}^{(1)}(x ; t)\right), \quad x>0 .
$$

Proof. The corollary follows if we show that

$$
F_{S}(x ; t)=\mathrm{e}^{t \Lambda(s)} \int_{0}^{x}\left(F^{(1)}(z ; t)+\sum_{k=1}^{\lceil z / s\rceil-1}(-t)^{k} A_{s ; k}^{(1)}(z ; t)\right) \mathrm{d} z .
$$

From (2.4), it is enough to prove that

$$
A_{s ; k}(x ; t)=\int_{k s}^{x} A_{s ; k}^{(1)}(z ; t) \mathrm{d} z, \quad x>k s .
$$

By virtue of (2.9) and its extension (2.10), the last equation reads

$$
\int_{k s}^{x} \int_{u}^{x} F^{(1)}(z-u ; t) \mathrm{d} z \lambda_{s}^{* k}(\mathrm{~d} u)=\int_{k s}^{x} \int_{k s}^{z} F^{(1)}(z-u ; t) \lambda_{s}^{* k}(\mathrm{~d} u) \mathrm{d} z
$$

where on the left-hand side we have used $F=\int F^{(1)}$ and $F(0 ; t)=0$, and follows from Tonelli's theorem. (Less elegantly, (2.18) can be proved by induction based on (2.8).) 
Remark 2.5. It is well known (cf., e.g. [17, Theorem 27.7]) that any Lévy process with infinite absolutely continuous Lévy measure possesses a density at each time $t>0$. Thus, Corollary 2.1 can be applied to a wide class of subordinators.

Remark 2.6. By simply considering (2.14) with domain of integration $C_{s ; k}(x)$ and measure $v_{\mid B}(\mathrm{~d} u)=\mathbf{1}_{B}(u) v(\mathrm{~d} u)$, we can now immediately deduce the analog of Corollary 2.1 for the density function of $X_{\backslash B}(t)$, denoted by $F_{\backslash B}^{(1)}(\cdot ; t)$. Specifically, under the assumption of Corollary 2.1, we have

$$
\begin{aligned}
F_{\backslash B}^{(1)}(x ; t) & \\
=\mathrm{e}^{t v(B)}\left(F^{(1)}(x ; t)+\sum_{k=1}^{\lceil x / s\rceil-1}(-t)^{k} \int_{C_{s ; k}(x) \cap B^{k}}\right. & F^{(1)}\left(x-\sum_{i=1}^{k} u_{i} ; t\right) \\
& \left.\times v\left(\mathrm{~d} u_{1}\right) \cdots v\left(\mathrm{~d} u_{k}\right)\right), \quad x>0 .
\end{aligned}
$$

In Proposition 2.1, below, we give a continuous variant of (2.17) for the derivatives $F_{s}^{(j)}(x ; t)$, under suitable regularity conditions on $F(\cdot ; t)$ and $v$. An elementary proof is sketched in Appendix A. We first recall the following standard notation and notion.

Let $C^{n}(I)$ for $n \in \mathbb{N} \cup\{\infty\}$ and $C^{0}(I)$ denote the sets of real-valued functions that are $n$ times continuously differentiable and, respectively, continuous on $I$.

A function $f$ is piecewise smooth on an interval $[a, b]$ if there exist $a=x_{1}<\cdots<x_{m}=b$ such that, for all $i=1, \ldots, m-1$, both $f$ and $f^{\prime}$ are continuous on $\left(x_{i}, x_{i+1}\right)$ with finite limits at the endpoints.

Proposition 2.1. Suppose that $F(\cdot ; t) \in C^{n-1}(\mathbb{R})$ and $F^{(n)}(\cdot ; t) \in C^{0}((0, \infty))$ for some $n \geq 1$. Furthermore, suppose that one of the following conditions is satisfied.

(i) $F^{(n)}(\cdot ; t)$ is bounded near $0^{+}$.

(ii) $F^{(n)}(\cdot ; t)$ is unbounded and absolutely integrable (with respect to the Lebesgue measure) near $0^{+} ; v$ is absolutely continuous with density $\rho$, which is bounded on $[s, b]$ for all $b>s$.

(iii) $F^{(n)}(\cdot ; t)$ is not absolutely integrable near $0^{+}$(hence unbounded there); $v$ is absolutely continuous with density $\rho$, which is piecewise smooth on $[s, b]$ for all $b>s$.

Then, $F_{s}(\cdot ; t) \in C^{n-1}(\mathbb{R}), F_{s}^{(n)}(\cdot ; t) \in C^{0}((0, \infty))$, and, for every $j=1, \ldots, n$,

$$
F_{S}^{(j)}(x ; t)=\mathrm{e}^{t \Lambda(s)}\left(F^{(j)}(x ; t)+\sum_{k=1}^{\lceil x / s\rceil-1}(-t)^{k} A_{s ; k}^{(j)}(x ; t)\right), \quad x>0 .
$$

The integrals corresponding to case (iii) with $j=n$ are interpreted as iterated (generally improper) Riemann integrals according to (2.3), setting $v\left(\mathrm{~d} u_{i}\right)=\rho\left(u_{i}\right) \mathrm{d} u_{i}$.

For simplicity of terminology, we did not consider weaker assumptions on $v$. The ones given above are quite satisfactory, and improvements can be made based on the sketched proof of this proposition. We also note that a function $F^{(n)}(\cdot ; t)$ as in Proposition 2.1(iii) is necessarily the difference of two nonnegative nonintegrable functions. 
Here it is worth mentioning the important class of pure-jump self-decomposable subordinators (see, e.g. [17]), which are characterized by an absolutely continuous Lévy measure of the form $v(\mathrm{~d} x)=k(x) / x \mathrm{~d} x, x>0$, where $k(x) \geq 0$ is monotone decreasing on $(0, \infty)$ (a priori satisfying the integrability condition $\left.\int_{0}^{\infty}(x \wedge 1)(k(x) / x) \mathrm{d} x<\infty\right)$. If $k\left(0^{+}\right)=\infty$ then $F(\cdot ; t) \in C^{\infty}(\mathbb{R})[17$, Theorem 28.4(ii)], the standard example being the stable subordinator (see Example 3.2, below). If $k\left(0^{+}\right)=c, 0<c<\infty$, then $F(\cdot ; t) \in C^{n-1}(\mathbb{R})$ and $F^{(n)}(\cdot ; t) \in C^{0}((0, \infty))$, where $n=\lceil c t\rceil$ (cf. [17, Remark 28.7]). The last fact is apparent for the gamma process, as defined in Section 4.1, below; a nonelementary example is provided by the subordinator with generalized Dickman (one-dimensional) marginals defined at the beginning of that subsection.

Remark 2.7. Reasoning as in Remark 2.6, we can easily deduce the analog of Proposition 2.1 for the derivatives $F_{\backslash B}^{(j)}(\cdot ; t), j=1, \ldots, n$. The result is analogous to (2.19). To be more precise, if $F^{(n)}(\cdot ; t)$ is as in Proposition 2.1(iii) then we assume that the function $\mathbf{1}_{B}(u) \rho(u)$ is piecewise smooth on $[s, b]$ for all $b>s$ and that the integrals associated with $F_{\backslash B}^{(n)}(x ; t)$ are iterated (possibly improper) Riemann integrals according to (2.3), incorporating the factor $\prod_{i=1}^{k} \mathbf{1}_{B}\left(u_{i}\right)$.

\section{Applications involving the ordered jumps}

In this section we shall use (2.4) and a simple generalization of its particular case (2.11) to derive various formulae which complete/generalize certain existing results on the ordered jumps of subordinators that can be found in [5], [14], or [15].

\subsection{General formulae}

As in Section 1 , let $V_{i}(t)$ for $i=1,2, \ldots$ be the ordered jumps in $(0, t]$ of a pure-jump subordinator $X$ (CPP or not) with continuous Lévy measure $v$ and marginal DF $F(\cdot ; t)$, and define $X^{b}=\{X(t)+b t: t \geq 0\}$, the associated subordinator with drift $b \geq 0$. Furthermore, define the ratio $R_{n}^{b}(t)$ for all $n \in \mathbb{N}$ as

$$
R_{n}^{b}(t)=\frac{V_{n}(t)}{X^{b}(t)-\sum_{i=1}^{n-1} V_{i}(t)}=\frac{V_{n}(t)}{\sum_{i=n}^{\infty} V_{i}(t)+b t},
$$

interpreting $0 / 0$ as 0 in the case where $V_{n}(t)=0$ and $b=0$. Now, if we let $M \in(0, \infty]$ denote the supremum of the support of $v$, we easily see that $M /(M+b t)$ is the supremum of the support of the RV $R_{n}^{b}(t)$. Here and later, we define $M /(k M+b t)=1 / k$ if $M=\infty$.

Using the notation above and the notation introduced in the preceding sections, we can state and prove the following proposition which follows easily from Theorem 2.1 and provides, together with the corollary that follows it, a substantial extension of Equation (8) of [14] (restated below as (3.4)).

Proposition 3.1. For every $u \in(0, M /(M+b t))$, the tail distribution function of $R_{n}^{b}(t)$ at $u$ is given by

$$
\begin{aligned}
& \mathrm{P}\left(R_{n}^{b}(t)>u\right) \\
& =\frac{t^{n}}{(n-1) !} \int_{0}^{M}( \\
& \quad F\left(\frac{1-u}{u} s-b t ; t\right) \\
& \left.\quad+\sum_{k=1}^{\lceil 1 / u-b t / s\rceil-2}(-t)^{k} A_{s ; k}\left(\frac{1-u}{u} s-b t ; t\right)\right) \Lambda(s)^{n-1} v(\mathrm{~d} s) .
\end{aligned}
$$


Its distribution has mass $\sum_{i=0}^{n-1} P_{i}(t \Lambda(0))$ at 0 if $X$ is a CPP and mass $P_{n}(t \Lambda(0))$ at 1 if, in addition, $b=0$. In any other case the mass is 0 .

Proof. Fix $u \in(0, M /(M+b t))$. Recall from Section 1 that $\sum_{i=n+1}^{\infty} V_{i}(t)$ conditioned on $V_{n}(t)=s, s>0$, is distributed like $X_{s}(t)$. Thus, using the law of total probability and recalling the notation in (1.2), we can write

$$
\mathrm{P}\left(R_{n}^{b}(t)>u\right)=0 \cdot \mathrm{P}\left(V_{n}(t)=0\right)+\int_{0^{+}}^{M} \mathrm{P}\left(X_{s}(t)<\frac{1-u}{u} s-b t\right) P_{V_{n}(t)}(\mathrm{d} s) .
$$

Equation (3.2) now follows straightforwardly by substitution of (1.2) and (2.4) (noting that $\lceil\xi-1\rceil=\lceil\xi\rceil-1)$. The last part of the proposition is very easy to check.

We note that if $M=\infty$ but $\Lambda(s)$ decreases to 0 rapidly as $s \rightarrow \infty$, v may be truncated considerably from above, depending on the accuracy we wish to achieve, thus making (3.2) more practical for numerical computations. If $v$ is absolutely continuous then (3.3), below, may be a good alternative to (3.2).

We also note the following observation:

$$
\mathrm{P}\left(V_{n}(t)>y\left(\sum_{i=n+1}^{\infty} V_{i}(t)+b t\right)\right)=\mathrm{P}\left(R_{n}^{b}(t)>\frac{y}{y+1}\right), \quad y>0 .
$$

In view of the next subsection we note that a relation like this also holds if the infinite series are truncated at some integer $m>n$.

Corollary 3.1. Suppose that $v$ is absolutely continuous with density $\rho$. Then, for every $b \geq 0$ and $y \in(b t / M, \infty)$,

$$
\begin{aligned}
\mathrm{P}\left(R_{n}^{b}(t)>\frac{1}{1+y}\right)=\frac{t^{n}}{(n-1) !} \sum_{k=0}^{\lceil y\rceil-1}( & -t)^{k} \int_{C_{1 ; k}(y)} \int_{0}^{M} F\left(\left(y-\sum_{i=1}^{k} u_{i}\right) s-b t ; t\right) \\
& \times\left(\prod_{i=1}^{k} \rho\left(u_{i} s\right)\right) s^{k} \Lambda(s)^{n-1} \rho(s) \mathrm{d} s \mathrm{~d} u_{1} \cdots \mathrm{d} u_{k} .
\end{aligned}
$$

Proof. We first set $u=1 /(1+y)$ in Proposition 3.1. Recalling that $\int_{C_{1 ; 0}(y)} \cdot=\cdot$, we then have to handle the expression

$$
\sum_{k=1}^{\lceil y-b t / s\rceil-1}(-t)^{k} \int_{C_{s ; k}(y s-b t)} F\left(y s-b t-\sum_{i=1}^{k} u_{i} ; t\right) v\left(\mathrm{~d} u_{1}\right) \cdots v\left(\mathrm{~d} u_{k}\right) .
$$

Since $F\left(y s-b t-\sum_{i=1}^{k} u_{i} ; t\right)$ is 0 when $\sum_{i=1}^{k} u_{i}>y s-b t$, it follows that we can set the term $b t$ to 0 in both $\lceil y-b t / s\rceil-1$ and $C_{s ; k}(y s-b t)$ above. We then make an iterative change of variable $u_{i} / s \mapsto u_{i}, i=1, \ldots, k$, corresponding to (2.3). The proof is completed by applying Tonelli's theorem.

Perman [14], whose work has considerably influenced the present paper, derived in a different way a similar result to our Corollary 3.1 in the case $n=1$ and $b=0$. Specifically, it is given, for every $u \in(0,1)$, by the following formula:

$$
\mathrm{P}\left(R_{1}^{0}(1)>u, X(1) \in \mathrm{d} s\right)=\sum_{k=1}^{\infty} \frac{(-1)^{k+1}}{k !} \int_{B_{k}^{u}} s^{k}\left(\prod_{i=1}^{k} h\left(y_{i} s\right)\right) f_{X(1)}\left(s \hat{y}_{k}\right) \prod_{i=1}^{k} \mathrm{~d} y_{i} \mathrm{~d} s,
$$


where, incorporating Perman's notation: $B_{k}^{u}=\left\{\left(u_{1}, \ldots, u_{k}\right): u<u_{i}<1, \sum_{i} u_{i}<1\right\}$ (thus the sum is finite); the Lévy measure is assumed infinite and absolutely continuous with density $h$; $f_{X(\cdot)}$ is the density of the $\mathrm{RV} X(\cdot)$ (recall Remark 2.5 for its existence); and $\hat{y}_{k}=1-\sum_{i=1}^{k} y_{i}$. The tail DF of $R_{1}^{0}(1)$ is computed by integration over $s$.

Whether to use (3.3) or (3.4) to obtain the tail DF of $R_{1}^{0}(\cdot)$ depends on the specific problem. The primary advantage of (3.3) is, of course, its generality. Equation (3.2) adds the ability to handle the case where $v$ is only assumed to be continuous. In the next subsection we shall examine a special case in which our kind of representation is particularly fruitful.

Perman [14, Theorem 2] also found a formula for the $(n+1)$-dimensional joint density $p_{n}\left(s, y_{1}, \ldots, y_{n}\right)$ of the random vector $\left(X(1), V_{1}(1) / X(1), \ldots, V_{n}(1) / X(1)\right)$, again restricted to a drift-free subordinator with infinite Lévy measure which is absolutely continuous with density $h$. The formula uses the integral equation $p_{1}(s, y)=s h(s y) \int_{0}^{(y /(1-y)) \wedge 1} p_{1}(s(1-y), v) \mathrm{d} v$. This integral equation was later used in [15, Proposition 45] to obtain the formula $p_{1}(s, y)=$ $\sum_{k=1}^{\infty}(-1)^{k+1} f_{k}(s, y)$, where the $f_{k}$ s are functions defined recursively in some manner and all but at most the first $k$ terms of the sum are 0 if $y>1 /(k+1)$. Furthermore, on integrating the last equation with respect to $y$ from $u$ to 1 we obtain a series expansion for $\mathrm{P}\left(X(1) \in \mathrm{d} s, V_{1}(1) / X(1)>u\right)$, which, according to [15, Remark 46], can be shown to be identical to that obtained by Perman in (3.4).

Example 3.1. Let $\gamma(a, b)$ denote the lower incomplete gamma function (as defined in Example 2.1). Thanks to a particular case of Equations (6) and (8) in [10], involving the hypergeometric function ${ }_{2} F_{1}$, we have

$$
\int_{0}^{\infty} \mathrm{e}^{-\omega s} s^{-1} \gamma(q, s) \mathrm{d} s=\Gamma(q) \sum_{j=0}^{\infty} \frac{1}{q+j} \frac{1}{(1+\omega)^{q+j}}, \quad \omega>0, q>0 .
$$

Alternatively, we can obtain (3.5) in a more elementary way through differentiating both sides with respect to $\omega$. Now let $X$ be a $\Gamma_{c, \lambda}$ process as defined in Section 4.1, below. It follows from (3.3) and (3.5), after elementary substitutions, that, for every $y \in(0, \infty)$,

$$
\begin{aligned}
& \mathrm{P}\left(R_{1}^{0}(t)>\frac{1}{1+y}\right) \\
& \quad=c t \sum_{k=0}^{\lceil y\rceil-1}(-c t)^{k} \int_{C_{1 ; k}(y)} \sum_{j=0}^{\infty} \frac{1}{c t+j}\left(\frac{y-\sum_{i=1}^{k} u_{i}}{y+1}\right)^{c t+j} \frac{\mathrm{d} u_{1} \cdots \mathrm{d} u_{k}}{u_{1} \cdots u_{k}} .
\end{aligned}
$$

(The scaling property of gamma processes, $\Gamma_{c, \lambda} \stackrel{\text { LAW }}{=} \lambda^{-1} \Gamma_{c, 1}$, accounts for the independence on $\lambda$.) We note that the infinite series in (3.6) can be replaced by integrals according to the identity $\sum_{j=0}^{\infty} \xi^{c t+j}(c t+j)^{-1}=\int_{0}^{\xi}(1-z)^{-1} z^{c t-1} \mathrm{~d} z, \xi \in(0,1)$, which follows straightforwardly from term-by-term integration. Actually, a slightly different version of (3.6) was obtained by Perman, in a simpler manner, via (3.4). The use of infinite series in (3.6) may give a little advantage to our equation. We also note that using the Laplace transform (3.5), the following formula is easily verified:

$$
\mathrm{E}\left[E_{1}(Y)\right]=\sum_{j=0}^{\infty}\left(\frac{\lambda}{\lambda+1}\right)^{t+j} \frac{1}{t+j}=\int_{0}^{\lambda /(\lambda+1)} \frac{z^{t-1}}{1-z} \mathrm{~d} z
$$

where $E_{1}(x)=\int_{x}^{\infty} y^{-1} \mathrm{e}^{-y} \mathrm{~d} y$ is the exponential integral function and $Y$ is a gamma $(t, \lambda) \mathrm{RV}$ (that is, $Y \stackrel{\text { LAW }}{=} X(t), X$ being a $\Gamma_{1, \lambda}$ process). 
The distribution of $R_{1}^{0}(t)$ in the case where $X$ is a gamma process is well studied in probability theory. We recall from [9] that the Poisson-Dirichlet distribution with parameter $\theta$, abbreviated as $\operatorname{PD}(\theta)$, is the distribution of the infinite vector $\left(V_{1}(\theta) / X(\theta), V_{2}(\theta) / X(\theta), \ldots\right)$, where $X$ is a $\Gamma_{1,1}$ process. It follows that if $X$ is a $\Gamma_{c, \lambda}$ process then $R_{1}^{0}(t)$ is distributed as the largest component of the $\operatorname{PD}(c t)$ distribution (recall the above example for the independence on $\lambda$ ). The Poisson-Dirichlet distribution will be briefly encountered in the next section.

The next example provides a generalization of a result given by Perman for the tail DF of $V_{1}(1) / X(1)$ in the case where $X$ is a stable subordinator.

Example 3.2. Let $X$ be a strictly $\alpha$-stable subordinator, $0<\alpha<1$. Then (see, e.g. [15]) $\Lambda(x)=c x^{-\alpha}, x>0$, for some constant $c>0$. The corresponding Lévy density is $\rho(x)=$ $c \alpha x^{-\alpha-1} \mathbf{1}_{(0, \infty)}(x)$. Since the distribution of $R_{n}^{0}(t)$ in this case is independent of time $t$ (see Example 3.4, below), we shall assume for the moment that $t=1$. The following formula for certain negative moments of $X(1)$ will play a key role in the derivation of our result:

$$
\mathrm{E}\left[X(1)^{-m \alpha}\right]=\frac{m !}{c^{m}} \frac{1}{[\Gamma(1-\alpha)]^{m} \Gamma(m \alpha+1)}, \quad m \in \mathbb{N} .
$$

This formula is obtained by substitution of $m \alpha$ into Equation (160) of [15] or Equation (19) of [14] for the negative moments of $X(1)$, where the constant $c_{\alpha}$ appearing in the latter equation should be regarded as $c \alpha$. The negative moments in (3.7) for $m=n+k, k=0,1,2, \ldots$, appear when applying (3.3), using integration by parts with respect to $s$, to the computation of the tail DF of $R_{n}^{0}(1)$. Recalling the independence on $t$, we then find that, for every $y \in(0, \infty)$,

$$
\begin{aligned}
\mathrm{P}\left(R_{n}^{0}(t)>\frac{1}{1+y}\right)= & \sum_{k=0}^{\lceil y\rceil-1}(-\alpha)^{k} \frac{(n+k-1) !}{(n-1) !} \frac{1}{[\Gamma(1-\alpha)]^{n+k} \Gamma((n+k) \alpha+1)} \\
& \times \int_{C_{1 ; k}(y)} \frac{\left(y-\sum_{i=1}^{k} u_{i}\right)^{(n+k) \alpha}}{\left(u_{1} \cdots u_{k}\right)^{\alpha+1}} \mathrm{~d} u_{1} \cdots \mathrm{d} u_{k} .
\end{aligned}
$$

Here we note that, by applying (3.4) and using [14, Equation (19)], Perman derived a slightly different version of (3.8) for the case in which $n=1$. (Actually, the powers of $\Gamma(1-\alpha)$ appearing in his result should be increased by 1.) It should also be noted (cf. [15, Propositions 10 and 11] or [5, Proposition 2]) that the ratio $\Sigma_{n}(t):=\sum_{i=n+1}^{\infty} V_{i}(t) / V_{n}(t)$, whose DF satisfies $\mathrm{P}\left(\Sigma_{n}(t) \leq y\right)=\mathrm{P}\left(R_{n}^{0}(t)>1 /(1+y)\right), y \in(0, \infty)$, is distributed as the sum of $n$ independent copies of $\Sigma_{1}(t)$, which in turn admits the Laplace transform $\mathrm{E}\left[\exp \left[-\omega \Sigma_{1}(t)\right]\right]=$ $\psi_{\alpha}(\omega)^{-1}, \omega \geq 0$, where $\psi_{\alpha}(\omega)=1+\alpha \int_{0}^{1}\left(1-\mathrm{e}^{-\omega x}\right) x^{-\alpha-1} \mathrm{~d} x$. A partial result for the DF of $\sum_{i=n+1}^{m} V_{i}(t) / V_{n}(t)$ for all $m>n$ will be given in Example 3.4, below.

Remark 3.1. From (3.8) we can easily obtain the density function, say $q_{n}$, of $R_{n}^{0}(t)$. Indeed, noting that the function $\varphi(y)=y^{(n+k) \alpha} \mathbf{1}_{[0, \infty)}(y)$ belongs to $C^{0}(\mathbb{R})$ with $\varphi(0)=0$, we deduce from the sketched proof of Proposition 2.1 that we can differentiate the right-hand side of (3.8) under the integral sign and, thus, show that, for every $y \in(0, \infty)$,

$$
\begin{aligned}
q_{n}\left(\frac{1}{1+y}\right) \frac{1}{(1+y)^{2}}= & \sum_{k=0}^{\lceil y\rceil-1}(-1)^{k} \alpha^{k+1} \frac{(n+k) !}{(n-1) !} \frac{1}{[\Gamma(1-\alpha)]^{n+k} \Gamma((n+k) \alpha+1)} \\
& \times \int_{C_{1 ; k}(y)} \frac{\left(y-\sum_{i=1}^{k} u_{i}\right)^{(n+k) \alpha-1}}{\left(u_{1} \cdots u_{k}\right)^{\alpha+1}} \mathrm{~d} u_{1} \cdots \mathrm{d} u_{k} .
\end{aligned}
$$


Note that the density function of $\Sigma_{n}(t)$, and, hence, the inverse Laplace transform of $\psi_{\alpha}(\omega)^{-n}$, is given by the right-hand side of (3.9).

\subsection{A special tractable case}

Restricting $u$ in Proposition 3.1 to the domain in which the sum appearing on the right-hand side of (3.2) vanishes, we obtain a particularly convenient and useful case of this proposition, which can even be easily generalized in some manner. Before we begin our investigation, we need the following notation and observation. For $n \in \mathbb{N} \cup\{\infty\}$, we define the value of the operator $K_{n ; t}$ acting on an arbitrary subordinator $Z$ to be the sum of the $n$ largest jumps of $Z$ in the time interval $(0, t]$. Thus, in particular, $K_{\infty ; t}(Z)=Z(t)$. Following the first step in the proof of Theorem 2.1 with $K_{n ; t}$ operating on $X$ and $X_{s}$, we see that

$$
\mathrm{P}\left(K_{n ; t}\left(X_{s}\right) \leq x\right) \mathrm{e}^{-t \Lambda(s)}=\mathrm{P}\left(K_{n ; t}(X) \leq x\right) \text { for all } x \leq s .
$$

Now, in a similar manner to Proposition 3.1, and using the same notation and conventions, the following result can be established.

Proposition 3.2. For any $n<m \leq \infty, b \geq 0$, and $u \in[M /(2 M+b t), M /(M+b t))$,

$$
\begin{aligned}
& \mathrm{P}\left(\frac{V_{n}(t)}{\sum_{i=n}^{m} V_{i}(t)+b t}>u\right) \\
& \quad=\frac{t^{n}}{(n-1) !} \int_{0}^{M} \mathrm{P}\left(\sum_{i=1}^{m-n} V_{i}(t) \leq \frac{1-u}{u} s-b t\right) \Lambda(s)^{n-1} v(\mathrm{~d} s) .
\end{aligned}
$$

Proof. First note that, conditioned on $V_{n}(t)=s, s>0$, the RV $\sum_{i=n+1}^{m} V_{i}(t)$ is distributed as $K_{m-n ; t}\left(X_{s}\right)$. Then follow the proof of Proposition 3.1, noting that $u \geq M /(2 M+b t)$ implies that $(1-u) u^{-1} s-b t \leq s$ for all $s \in(0, M]$, and apply (3.10).

Let us now mention two particularly interesting cases of Proposition 3.2. Setting $m=\infty$, $b=0$, and $u=\frac{1}{2}$ in (3.11), we obtain

$$
\mathrm{P}\left(V_{n}(t)>V_{n+1}(t)+V_{n+2}(t)+\cdots\right)=\frac{t^{n}}{(n-1) !} \int_{0}^{M} F(s ; t) \Lambda(s)^{n-1} v(\mathrm{~d} s)
$$

(more generally, note the formula for $\left.\mathrm{P}\left(V_{n}(t)>\sum_{i=n+1}^{m} V_{i}(t)\right), m \leq \infty\right)$, and setting $m=\infty$, $n=1$, and $u=\frac{1}{2}$, we obtain

$$
\mathrm{P}\left(V_{1}(t)>\frac{X^{b}(t)}{2}\right)=t \int_{0}^{M} \mathrm{P}\left(X^{b}(t) \leq s\right) v(\mathrm{~d} s), \quad b \geq 0
$$

(note that (3.11) remains valid also if $u=\frac{1}{2} \geq M /(M+b t)$ ). Moreover, if $X$ is a CPP with rate $\lambda$ and i.i.d. jumps $Y_{i}, i=1,2, \ldots$, then the right-hand side of (3.13) is none other than $\lambda t \mathrm{P}\left(X^{b}(t) \leq Y\right)$ or, equivalently, $\lambda t \mathrm{E}\left[F^{b}(Y ; t)\right]$, where $Y$ is independent of $X$ and distributed as $Y_{1}$ and $F^{b}(\cdot ; t)$ denotes the DF of $X^{b}(t)$.

We continue with the following elegant result analogous to Proposition 3.2.

Corollary 3.2. For any $n<m \leq \infty, b \geq 0$, and $u \in[M /(2 M+b t), M /(M+b t))$,

$$
\mathrm{P}\left(\frac{V_{n}(t)}{\sum_{i=n}^{m} V_{i}(t)+b t}>u\right)=\frac{t^{n}}{n !} \mathrm{E}\left[\left(\Lambda\left\{\frac{u}{1-u}\left(\sum_{i=1}^{m-n} V_{i}(t)+b t\right)\right\}\right)^{n}\right]
$$


Proof. Define $Z=(u /(1-u))\left(\sum_{i=1}^{m-n} V_{i}(t)+b t\right)$, and let $P_{Z}$ denote its distribution. Then,

$$
\begin{aligned}
\mathrm{E}\left[\Lambda(Z)^{n}\right] & =\int_{\{0\}} \Lambda(s)^{n} P_{Z}(\mathrm{~d} s)+\lim _{\varepsilon \rightarrow 0^{+}} \int_{\varepsilon}^{M} \Lambda(s)^{n} P_{Z}(\mathrm{~d} s) \\
& =\mathbf{1}_{(0, \infty)}(\Lambda(0)) \mathbf{1}_{\{0\}}(b) \Lambda(0)^{n} \mathrm{e}^{-t \Lambda(0)}+\lim _{\varepsilon \rightarrow 0^{+}} \int_{\varepsilon}^{M} \Lambda(s)^{n} \mathrm{dP}(Z \leq s) .
\end{aligned}
$$

By integration by parts for Stieltjes integrals we have

$$
\lim _{\varepsilon \rightarrow 0^{+}} \int_{\varepsilon}^{M} \Lambda(s)^{n} \mathrm{dP}(Z \leq s)=\lim _{\varepsilon \rightarrow 0^{+}}\left(\left.\Lambda(s)^{n} \mathrm{P}(Z \leq s)\right|_{\varepsilon} ^{M}-\int_{\varepsilon}^{M} \mathrm{P}(Z \leq s) \mathrm{d} \Lambda(s)^{n}\right) .
$$

We now note that if $\Lambda(0)=\infty$ then $\lim _{\varepsilon \rightarrow 0^{+}} \Lambda(\varepsilon)^{n} \mathrm{P}(Z \leq \varepsilon)=0$. Indeed, assuming the nontrivial case $b=0, Z \leq \varepsilon$ implies that $V_{1}(t) \leq \varepsilon$, and in turn $\mathrm{P}\left(V_{1}(t) \leq \varepsilon\right)=\mathrm{e}^{-t \Lambda(\varepsilon)}$. It follows that

$$
\mathrm{E}\left[\Lambda(Z)^{n}\right]=n \lim _{\varepsilon \rightarrow 0^{+}} \int_{\varepsilon}^{M} \mathrm{P}(Z \leq s) \Lambda(s)^{n-1} v(\mathrm{~d} s)
$$

Multiplying both sides of this equation by $t^{n} / n$ ! and applying Proposition 3.2, we arrive at (3.14).

The analog of (3.12) is given by the interesting formula

$$
\mathrm{P}\left(V_{n}(t)>V_{n+1}(t)+V_{n+2}(t)+\cdots\right)=\frac{t^{n}}{n !} \mathrm{E}\left[\Lambda(X(t))^{n}\right]
$$

(as before, note also the general case of $\left.\mathrm{P}\left(V_{n}(t)>\sum_{i=n+1}^{m} V_{i}(t)\right), m \leq \infty\right)$, while the analog of (3.13) is

$$
\mathrm{P}\left(V_{1}(t)>\frac{X^{b}(t)}{2}\right)=t \mathrm{E}\left[\Lambda\left(X^{b}(t)\right)\right], \quad b \geq 0 .
$$

This time we note that, for $\tilde{X}$ independent of $X$ and having the same law, $t \mathrm{E}\left[\Lambda\left(X^{b}(t)\right)\right]$ is none other than the expected number of jumps in $(0, t]$ of the process $\tilde{X}$ whose size exceeds $X^{b}(t)$.

Examples 3.3 and 3.4, below, provide simple applications of Corollary 3.2.

Example 3.3. Let $X^{b}$ be a CPP with exponential jumps as in Example 2.1 and drift $b \geq 0$. It follows from $(1.1)$ that $\mathrm{E}\left[\exp \left[-\omega X^{b}(t)\right]\right]=\exp [-\lambda t(\omega /(\omega+\theta))-b t \omega], \omega \geq 0$. From this, together with $\Lambda(x)=\lambda \mathrm{e}^{-\theta x}$ and (3.14), it follows straightforwardly that

$$
\mathrm{P}\left(R_{n}^{b}(t)>u\right)=\frac{(\lambda t)^{n}}{n !} \exp \left[-\lambda t \frac{n u}{(n-1) u+1}-b t \theta \frac{n u}{1-u}\right], \quad u \in\left[\frac{1}{2}, 1\right] .
$$

We note that since the exponential $(\theta)$ law is the same as that of a standard exponential RV divided by $\theta, R_{n}^{0}(t)$ is independent of $\theta$.

Example 3.4. Let $X^{b}$ be an $\alpha$-stable subordinator, $0<\alpha<1$, with drift $b \geq 0$. Since $\Lambda(x)=c x^{-\alpha}$, we have, by (3.14), for any $n<m \leq \infty$ and $u \in\left[\frac{1}{2}, 1\right)$,

$$
\mathrm{P}\left(\frac{V_{n}(t)}{\sum_{i=n}^{m} V_{i}(t)+b t}>u\right)=\frac{(c t)^{n}}{n !} \mathrm{E}\left[\left(\sum_{i=1}^{m-n} V_{i}(t)+b t\right)^{-n \alpha}\right]\left(\frac{1-u}{u}\right)^{n \alpha} .
$$

We recall from [17, Chapter 3] that the process $X$ (i.e. the jump part of $X^{b}$ ) in this case is self-similar, and specifically, $\{X(a t): t \geq 0\} \stackrel{\mathrm{LAW}}{=}\left\{a^{1 / \alpha} X(t): t \geq 0\right\}$ for any $a>0$. It follows 
that, in particular, the ratio $V_{n}(t) / \sum_{i=n}^{m} V_{i}(t)$ is independent of time $t$, and, hence, of $c$ as well. In turn, this together with (3.15) implies that the DF of $\sum_{i=n+1}^{m} V_{i}(t) / V_{n}(t)$, say $H_{\alpha, n, m}$, is given on $[0,1]$ by

$$
H_{\alpha, n, m}(y)=\frac{1}{n !} \mathrm{E}\left[\left(\sum_{i=1}^{m-n} V_{i}(1)\right)^{-n \alpha}\right] y^{n \alpha}=\mathrm{P}\left(V_{n}(1)>\sum_{i=n+1}^{m} V_{i}(1)\right) y^{n \alpha} .
$$

The coefficients of $y^{n \alpha}$ can be easily simulated based on the iterative procedure in [5] mentioned in the next section.

Clearly, the support of $H_{\alpha, n, m}$ is $[0, m-n]\left(\mathbb{R}_{+}\right.$if $\left.m=\infty\right)$. Letting $m=n+1$, we thus see that $V_{n+1}(t) / V_{n}(t)$ has a beta $(n \alpha, 1)$ distribution, which we already know from [5] or [15]. Both references also indicate that the ratios $V_{n+1}(t) / V_{n}(t)$ are mutually independent. We refer to [5] for the generalization to the vector $\left(V_{n_{k+1}}(t) / V_{n_{k}}(t) ; k=1, \ldots, K\right), n_{1}<\cdots<n_{K}<n_{K+1}$.

\section{Gamma's small jumps and the generalized Dickman distribution revisited}

\subsection{Some preliminaries and a limit theorem}

Henceforth, let $X_{\star}^{c}$ (for $c>0$ fixed) denote the pure-jump subordinator whose Lévy density is $c x^{-1} \mathbf{1}_{(0,1]}(x)$. The Laplace transform of $X_{\star}^{c}(t)$ is thus given by

$$
\mathrm{E}\left[\exp \left[-\omega X_{\star}^{c}(t)\right]\right]=\exp \left[c t \int_{0}^{1} \frac{\mathrm{e}^{-\omega x-1}}{x} \mathrm{~d} x\right], \quad \omega \geq 0 .
$$

Clearly, $X_{\star}^{c}(t) \stackrel{\text { LAW }}{=} X_{\star}^{1}(c t)$. Following [13], we say that a RV $Z$ has a generalized Dickman distribution with shape parameter $\theta>0(Z \sim \operatorname{GD}(\theta)$ for short $)$ if it satisfies the fixed-point equation

$$
Z \stackrel{\text { LAW }}{=} U^{1 / \theta}(1+Z)
$$

where $U$ is a uniform $(0,1) \mathrm{RV}$, independent of the $Z$ on the right. The unique solution in law of (4.2) is commonly given by

$$
Z=U_{1}^{1 / \theta}+\left(U_{1} U_{2}\right)^{1 / \theta}+\left(U_{1} U_{2} U_{3}\right)^{1 / \theta}+\cdots,
$$

where $U_{1}, U_{2}, \ldots$ is a sequence of independent uniform $(0,1) \mathrm{RVs}$. The Laplace transform $\mathrm{E}\left[\mathrm{e}^{-\omega Z}\right]$ is identical to the right-hand side of (4.1) provided that $c t=\theta$ (see, e.g. [13, Proposition 3]). Thus, in particular, $X_{\star}^{1}(\theta) \sim \operatorname{GD}(\theta)$.

The generalized Dickman distribution has been extensively studied in the literature. It occurs, among others, in number-theoretical [8], [20] and combinatorial [1] contexts. Many of the basic properties of the $\mathrm{GD}(\cdot)$ distribution can be found, e.g. in [1], [7], [8], [13], [18], [19, pp. 90-95], and [20]. Of special importance is the case in which $\theta=1$. The probability density function of $Z$ is then identical to $\mathrm{e}^{-\gamma} \rho$, where $\gamma$ is Euler's constant and $\rho$ is the celebrated Dickman function (for the definition and proof, see [13, Section 3]). For general $\theta>0$, the density of $Z$ also appears in connection with the Poisson-Dirichlet distribution with parameter $\theta$. In fact, the joint density of the first $r$ components of the $\operatorname{PD}(\theta)$ distribution, that is, the joint density of the vector $\left(V_{1}(\theta) / X(\theta), \ldots, V_{r}(\theta) / X(\theta)\right)$, where $X$ is a $\Gamma_{1,1}$ process, is nicely expressible in terms of $Z$ 's density (see, e.g. [7], and cf. [9]). See also Remark 4.2, below, with regard to the DF of the largest component.

The $\operatorname{GD}(\theta)$ distribution function will be studied in detail in the next subsection. In what follows we are going to reveal an important feature of the process $X_{\star}^{c}$ with regard to a certain class of subordinators, the gamma process being the most prominent representative. 
Suppose that we wish to approximate, e.g. for simulation purposes, some pure-jump subordinator $X$ with infinite (not necessarily continuous) Lévy measure $\pi$. The easiest way may be to simply ignore the small jumps not exceeding some tiny threshold $\varepsilon>0$ or replace them by their expected value $\mu(\varepsilon) t$, where $\mu(\varepsilon):=\mathrm{E}\left[X_{\varepsilon}(1)\right]=\int_{(0, \varepsilon]} x \pi(\mathrm{d} x)$. The remaining jumps are then those of a CPP, denoted by $X^{\varepsilon}$, with Lévy measure $\pi \mid\{x>\varepsilon\}$, and, thus, considered straightforward to handle. It is well known [2] that in case $\pi$ is continuous, a normal approximation of the small jumps can be applied if and only if $\lim _{\varepsilon \rightarrow 0}(\sigma(\varepsilon) / \varepsilon)=\infty$, where $\sigma^{2}(\varepsilon):=\operatorname{var}\left[X_{\varepsilon}(1)\right]=\int_{(0, \varepsilon]} x^{2} \pi(\mathrm{d} x)$. We then have $X(t) \cong \mu(\varepsilon) t+\sigma(\varepsilon) W(t)+X^{\varepsilon}(t)$, where $W$ is a standard Brownian motion independent of $X^{\varepsilon}$. (See [2] for a thorough account of the normal approximation of small jumps of arbitrary Lévy processes.)

For the rest of this subsection, let $X$ be a $\Gamma_{c, \lambda}$ process, meaning that $X$ is a pure-jump subordinator with Lévy density $\rho(x)=c x^{-1} \mathrm{e}^{-\lambda x} \mathbf{1}_{(0, \infty)}(x)$. Accordingly, the probability density function of $X(t)$, say $f(\cdot ; t)$, is given by

$$
f(x ; t)=\frac{\lambda^{c t} x^{c t-1} \mathrm{e}^{-\lambda x}}{\Gamma(c t)} \mathbf{1}_{(0, \infty)}(x) .
$$

To put it another way, $X(t) \sim \operatorname{gamma}(c t, \lambda)$. The parameter $c$ controls the rate of jump arrivals (just as in $X_{\star}^{c}$ ) and $\lambda$ inversely controls the jump size. As is well known, the normal approximation of small jumps does not hold in the gamma case (indeed, $\sigma(\varepsilon) \sim \varepsilon \sqrt{c / 2}$ as $\varepsilon \rightarrow 0$ ). Ignoring the small jumps or replacing them by their expected value (which is asymptotically $c t \varepsilon$ ) may be adequate here. However, we have the following simple yet illuminating result.

Proposition 4.1. Suppose that $\tilde{X}$ is a pure-jump subordinator with Lévy density $\rho$ satisfying

$$
\rho(x) \sim \frac{c}{x} \text { as } x \rightarrow 0^{+} .
$$

Then $\tilde{X}_{\varepsilon} / \varepsilon$ converges weakly to $X_{\star}^{c}$ as $\varepsilon \rightarrow 0$.

The weak convergence stated above is understood to mean the weak convergence of $\tilde{X}_{\varepsilon_{n}} / \varepsilon_{n}$ to $X_{\star}^{c}$ for any sequence $\varepsilon_{n} \searrow 0$, which in turn is understood in the space $D[0, \infty)$ of càdlàg functions equipped with the Skorohod J1 topology (for details, we refer the reader to [11] and reference 198 therein). The practical implication is that such a process $\tilde{X}$ can be approximated as

$$
\tilde{X} \cong \varepsilon X_{\star}^{c}+\tilde{X}^{\varepsilon},
$$

with the process $X_{\star}^{c}$ being independent of the CPP $\tilde{X}^{\varepsilon}$ with Lévy density $\rho \mathbf{1}_{\{x>\varepsilon\}}$. Since $\rho(x)=c x^{-1} \mathrm{e}^{-\lambda x} \mathbf{1}_{(0, \infty)}(x)$ satisfies (4.3), approximation (4.4) is in particular valid with respect to the $\Gamma_{c, \lambda}$ process.

Proof of Proposition 4.1. Let $\left\{\varepsilon_{n}\right\}$ be an arbitrary sequence of positive numbers converging to 0 . By the assumption on the Lévy density, $\varepsilon_{n} x \rho\left(\varepsilon_{n} x\right) \rightarrow c$ as $n \rightarrow \infty$ uniformly for $x$ in $(0,1)$. Thus, by changing variable and taking the limit, we find that

$$
\exp \left[\int_{0}^{\varepsilon_{n}}\left(\exp \left[-\frac{\omega}{\varepsilon_{n}} x\right]-1\right) \rho(x) \mathrm{d} x\right] \rightarrow \exp \left[\int_{0}^{1}\left(\mathrm{e}^{-\omega x}-1\right) \frac{c}{x} \mathrm{~d} x\right], \quad \omega \geq 0 .
$$

Noting that the left-hand side is actually the Laplace transform of $\tilde{X}_{\varepsilon_{n}}(1) / \varepsilon_{n}$, while the righthand side is that of $X_{\star}^{c}(1)$, we conclude by the well-known continuity theorem for Laplace 
transforms that $\tilde{X}_{\varepsilon_{n}}(1) / \varepsilon_{n}$ converges in distribution to $X_{\star}^{c}(1)$, which in turn implies that $\tilde{X}_{\varepsilon_{n}} / \varepsilon_{n}$ converges weakly to $X_{\star}^{c}$ (since weak convergence of Lévy processes reduces to weak convergence of the marginal distributions at $t=1$; see, e.g. [11, Corollary VII.3.6]).

Remark 4.1. A thorough characterization of the class of pure-jump subordinators $\tilde{X}$ such that $\tilde{X}_{\varepsilon} / \varepsilon$ converges weakly to $X_{\star}^{c}$ as $\varepsilon \rightarrow 0$ is provided by the author in [4]. It turns out [4] that the Dickman limit plays a central role in the context of approximating small jumps of subordinators.

The approximation (4.4) is quite simple and efficient in general. Indeed, $X_{\star}^{c}$ admits the following series representation in the time interval $[0, T]$ :

$$
X_{\star}^{c}(t)=\sum_{j=1}^{\infty}\left(U_{1} \cdots U_{j}\right)^{1 /(c T)} \mathbf{1}_{\left\{\hat{U}_{j} \leq t\right\}},
$$

where $\left\{U_{j}\right\}$ and $\left\{\hat{U}_{j}\right\}$ are independent sequences of i.i.d. uniform $(0,1)$ and i.i.d. uniform $(0, T)$ $\mathrm{RVs}$, respectively. This fact can be easily verified using the simple iterative procedure for constructing the entire sequence of jumps in decreasing order, described very simply in [5]. Moreover, since $\varepsilon$ is assumed to be small and since the above series converges rapidly (depending, of course, on $c T$ ), it is clear that we may truncate the series at some moderate number of terms. As for the CPP $\tilde{X}^{\varepsilon}$, it has rate $\int_{\varepsilon}^{\infty} \rho(x) \mathrm{d} x$, which is asymptotically $c \log (1 / \varepsilon)$ as $\varepsilon \rightarrow 0$ (this is a particular case of a proposition in [4]), and is, hence, relatively small in general. This is very advantageous from a computational point of view.

The high quality of the approximations $\tilde{X} \cong \varepsilon X_{\star}^{c}+\tilde{X}^{\varepsilon}$ and $\tilde{X}_{\varepsilon} \cong \varepsilon X_{\star}^{c}$ has been analyzed by the author in [4] for some special class of self-decomposable subordinators and the $\Gamma_{c, \lambda}$ process in particular (see, e.g. the next subsection with respect to the second approximation). We also note that an analogous approximation to (4.4) with respect to the well-known variance gamma process is now straightforward, as this process can be expressed as the difference of two independent gamma processes (see, e.g. [12]).

It should, however, be remarked that there are simple and efficient alternatives to (4.4) with respect to the particularly tractable gamma case. The most natural one is to use a random walk approximation as follows: $X(t) \cong \sum_{i=1}^{\lfloor t / h\rfloor} Y_{i}, t \in[0, T)$, where the $Y_{i} \mathrm{~s}$ are i.i.d. gamma $(c h, \lambda) \mathrm{RVs}$ and $h=T / N$, where $N$ is a large integer. (A drawback is that we cannot precisely identify the location and magnitude of the significant jumps.) Rosiński [16] presented four series representations of a gamma process. A very useful one is given by $X(t)=\sum_{j=1}^{\infty} \lambda^{-1} \exp \left[-\Gamma_{j} /(c T)\right] V_{j} \mathbf{1}_{\left\{U_{j} \leq t\right\}}, t \in[0, T]$, where $\left\{\Gamma_{j}\right\}$ are the arrival times in a Poisson process with rate $1,\left\{V_{j}\right\}$ are i.i.d. standard exponential RVs, and $\left\{U_{j}\right\}$ are i.i.d. uniform $(0, T) \mathrm{RVs}$, with the three sequences being independent of each other.

To close this subsection, we remark that an approximation of small jumps of a gamma (or variance gamma) process, similar to the one presented above, has already been identified in the literature; see [6] or reference 7 therein. However, as described by the author in [4], the advantages of our approach are evident.

\subsection{Distributional results}

We now give an explicit formula for the DF of the RV $X_{\varepsilon}(t) / \varepsilon$ where $X$ is a $\Gamma_{1, \lambda}$ process, leading to a revisiting of the generalized Dickman distribution. The choice $c=1$ does not restrict generality as $\tilde{X}_{\varepsilon}(t) \stackrel{\text { LAW }}{=} X_{\varepsilon}(c t), \tilde{X}$ being a $\Gamma_{c, \lambda}$ process. Throughout this subsection, $\gamma$ stands for Euler's constant. 
A straightforward application of Theorem 2.1, combined with the classical identity

$$
\int_{\xi}^{\infty} \frac{\mathrm{e}^{-y}}{y} \mathrm{~d} y=-\gamma-\ln \xi+\int_{0}^{\xi} \frac{1-\mathrm{e}^{-y}}{y} \mathrm{~d} y, \quad \xi>0,
$$

yields

$$
\begin{aligned}
\mathrm{P}\left(\frac{X_{\varepsilon}(t)}{\varepsilon} \leq x\right)= & \exp \left[t \int_{0}^{\lambda \varepsilon} \frac{1-\mathrm{e}^{-y}}{y} \mathrm{~d} y\right] \frac{\mathrm{e}^{-\gamma t}}{\Gamma(t+1)} \\
& \times \sum_{k=0}^{\lceil x\rceil-1}(-t)^{k} \int_{C_{1 ; k}(x)} \sum_{j=0}^{\infty} \frac{t(-\lambda \varepsilon)^{j}}{j !(t+j)}\left(x-\sum_{i=1}^{k} u_{i}\right)^{t+j} \\
& \times \exp \left[-\lambda \varepsilon \sum_{i=1}^{k} u_{i}\right] \frac{\mathrm{d} u_{1} \cdots \mathrm{d} u_{k}}{u_{1} \cdots u_{k}} .
\end{aligned}
$$

The infinite series in (4.6) arises from a series expansion of the lower incomplete gamma function, and is very advantageous from a computational point of view. In fact, since $\varepsilon$ is assumed small (say $\leq 10^{-3}$ ), the first three terms of the series can already yield very high accuracy. Moreover, if $t$ is integer valued then we can replace the infinite sum by a finite sum, according to the identity

$$
\sum_{j=0}^{\infty} \frac{t(-\lambda \varepsilon)^{j}}{j !(t+j)} \xi^{t+j}=\frac{t !}{(\lambda \varepsilon)^{t}}\left(1-\mathrm{e}^{-\lambda \varepsilon \xi} \sum_{j=0}^{t-1} \frac{(\lambda \varepsilon \xi)^{j}}{j !}\right), \quad \xi>0, t=1,2, \ldots,
$$

where we have used (2.15). (Here, the order of magnitude of $\varepsilon$ has no significant role.)

Now let $H_{\theta}(\cdot)$ denote the $\operatorname{GD}(\theta)$ DF and, moreover, let $H_{\theta}^{(j)}(\cdot)$ denote its $j$ th derivative, where the $j=0$ case corresponds to $H_{\theta}(\cdot)$. From the previous subsection we have $X_{\varepsilon}(\theta) / \varepsilon \stackrel{\text { LAW }}{\longrightarrow} X_{\star}^{1}(\theta)$ as $\varepsilon \rightarrow 0$ and, in turn, $\mathrm{P}\left(X_{\varepsilon}(\theta) / \varepsilon \leq x\right) \rightarrow H_{\theta}(x)$ (for all $x \in \mathbb{R}$ as $H_{\theta}$ is continuous). Letting $\varepsilon \rightarrow 0$ in (4.6) and considering the sketched proof of Proposition 2.1 , noting that the function $x^{\theta} \mathbf{1}_{[0, \infty)}(x)$ belongs to $C^{\lceil\theta\rceil-1}(\mathbb{R})$, the following result can be established.

Proposition 4.2. The $\mathrm{GD}(\theta)$ distribution function $H_{\theta}(\cdot)$ is of class $C^{\lceil\theta\rceil-1}(\mathbb{R})$, its $\lceil\theta\rceil$ th derivative $H_{\theta}^{(\lceil\theta\rceil)}(\cdot)$ is of class $C^{0}((0, \infty))$, and, for $j=0,1, \ldots,\lceil\theta\rceil$,

$$
\begin{aligned}
H_{\theta}^{(j)}(x)= & \frac{\mathrm{e}^{-\gamma \theta}}{\Gamma(\theta+1-j)} \\
& \times\left(x^{\theta-j}+\sum_{k=1}^{\lceil x\rceil-1}(-\theta)^{k} \int_{C_{1 ; k}(x)}\left(x-\sum_{i=1}^{k} u_{i}\right)^{\theta-j} \frac{\mathrm{d} u_{1} \cdots \mathrm{d} u_{k}}{u_{1} \cdots u_{k}}\right), \quad x>0 .
\end{aligned}
$$

Note that the regularity of $H_{\theta}(\cdot)$ in Proposition 4.2 is actually indicated in Section 2, just before Remark 2.7.

Proposition 4.2 invites comparison with similar results in the literature. Before we consider some of the relevant references, let us make the following comment.

It follows readily from (4.6) and (4.7) that, for any fixed $x>0$ and $t>0$,

$$
\mathrm{P}\left(\frac{X_{\varepsilon}(t)}{\varepsilon} \leq x\right)-\mathrm{P}\left(X_{\star}^{1}(t) \leq x\right)=b(x, t) \lambda \varepsilon+O\left(\varepsilon^{2}\right) \quad \text { as } \varepsilon \rightarrow 0,
$$


where $b(x, t)$ is some nonnegative function. The nonnegativity of $b(x, t)$ is due to the fact that the $\mathrm{RV} X_{\star}^{1}(t)$ is stochastically larger than $X_{\varepsilon}(t) / \varepsilon$, which follows by comparing the corresponding Lévy densities $x^{-1} \mathbf{1}_{(0,1]}(x)$ and $\mathrm{e}^{-\lambda \varepsilon x} x^{-1} \mathbf{1}_{(0,1]}(x)$. In fact, as is shown by the author in [4] using a completely different approach, $b(x, t)=t \mathrm{P}\left(x-U \leq X_{\star}^{1}(t) \leq x\right)$, where $U$ is a uniform $(0,1) \mathrm{RV}$ independent of $X_{\star}^{1}(t)$, and (4.8) holds uniformly in $x>0$. Moreover, numerical results in [4], based on Monte Carlo simulations, indicate that the function $\psi(t):=\sup _{x} b(x, t)$ can be approximated rather well by the function $\sqrt{t /(4 \pi)}$ - which turns out to be its asymptotic limit as $t \rightarrow \infty$-starting from a relatively very small value of $t$, say $t=0.25$. An exact expression for the $O\left(\varepsilon^{2}\right)$ term in (4.8) can be found in [4].

Vervaat $[19$, p. 90] gave a formula for the GD density function which is essentially identical to the right-hand side of (4.7) with $j=1$, except that the sets $C_{1 ; k}(x)$ are replaced by the sets $D_{1 ; k}(x)$ and, accordingly, a multiplying factor of $1 / k$ ! is added. Our Proposition 4.2 was actually inspired by his result. See also [1, Lemma 4.7] (and cf. [9, pp. 6-7]) for a slight modification of Vervaat's formula. Without going into any details, we note that our result originated from a completely different approach.

In view of Remark 2.1 and (2.10), we can conclude the following analog of (4.7):

$$
H_{\theta}^{(j)}(x)=\frac{\mathrm{e}^{-\gamma \theta}}{\Gamma(\theta+1-j)}\left(x^{\theta-j}+\sum_{k=1}^{\lceil x\rceil-1} \frac{(-\theta)^{k}}{k !} \int_{k}^{x}(x-u)^{\theta-j} f^{* k}(u) \mathrm{d} u\right), \quad x>0,
$$

where $f^{* k}$ denotes the $k$-fold convolution of $f(y)=y^{-1} \mathbf{1}_{(1, \infty)}(y)$, which can be obtained recursively by $f^{* k}(y)=\int_{1}^{y-(k-1)} f^{*(k-1)}(y-z) z^{-1} \mathrm{~d} z(k=2,3, \ldots)$. Actually, the cases $j=0$ and $j=1$ are indicated in [18, pp. 376-377] and [7, pp. 341-342], respectively. It may be useful to note/recall in relation to (4.10), below, that the functions $f^{* k}(y)$ are serviceable for calculating $H_{\theta}^{(j)}(x)$ for all $j=0,1, \ldots,\lceil\theta\rceil$.

In his Theorem 1, Griffiths [7] gave a recursive algorithm useful for numerical computation of the $\operatorname{GD}(\theta)$ density function. By comparison with (4.7) or (4.9), the following generalization to arbitrary $j=0,1, \ldots,\lceil\theta\rceil$ is evident:

$$
H_{\theta}^{(j)}(x)=\frac{\mathrm{e}^{-\gamma \theta} x^{\theta-j}}{\Gamma(\theta+1-j)}\left(1+\sum_{k=1}^{\lceil x\rceil-1}(-\theta)^{k} K_{k}\left(\frac{x-k}{x-k+1} ; \theta-j\right)\right), \quad x>0,
$$

where $K_{k}(v ; \theta-1)=v^{\theta+k-1} \sum_{j=0}^{\infty} a_{k j} v^{j}, 0<v<1$; the coefficients $\left\{a_{k j}\right\}$ (which depend on $\theta$ ) are defined recursively in the theorem in a manner convenient for numerical implementation, yet quite complicated in appearance. We thus see that Griffiths' efficient (and hard to derive) algorithm for the GD density function can also be applied with no additional effort to the computation of the corresponding DF or the higher-order derivatives.

Remark 4.2. Writing the $\operatorname{GD}(\theta)$ density function as $\mathrm{e}^{-\gamma \theta} x^{\theta-1} \rho_{\theta}(x) / \Gamma(\theta)$, the function $\rho_{\theta}(x)$ is a generalization of Dickman's function $\rho(x)=\rho_{1}(x)$ already mentioned above (see [13, Section 3.4]). In fact, the largest component of the $\operatorname{PD}(\theta)$ distribution has $\mathrm{DF} \rho_{\theta}(1 / x), x>0$ (see [9] and [13]).

It should be noted, however, that an elegant relation between the functions $H_{\theta}^{(j)}(x)$ analogous to those given above is indicated in [20, p. 498], where the function $p(u)=p(u, a, b)$ corresponds to $H_{\theta}^{(j)}(x)(0 \leq j \leq\lceil\theta\rceil)$ when setting $u=x, a=j-\theta$, and $b=\theta$. The function $p(u)$ is defined as a particular solution of some differential-difference equation that 

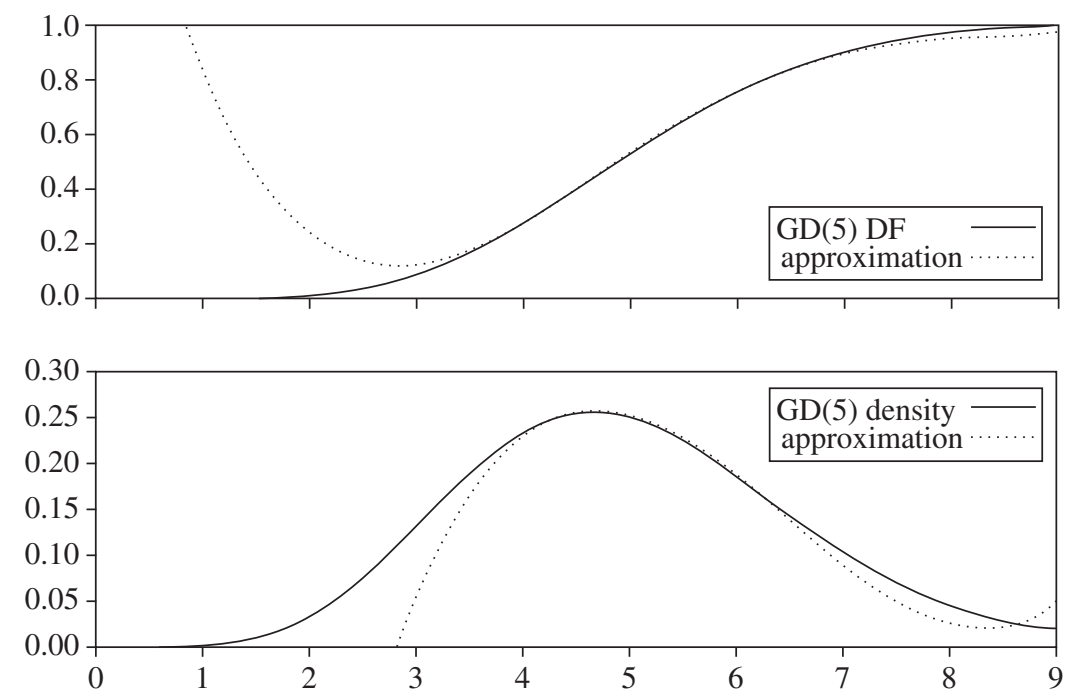

FIgURE 1: A fourth-order Taylor approximation to the GD(5) DF and a third-order Taylor approximation to the $\mathrm{GD}(5)$ density around 5 .

generalizes the well-known one corresponding to the GD density function (see, e.g. [1], [13], and [19, p. 90]). Moreover, an expression for $p(u)$ in [20, p. 497] gives, for $j=0,1, \ldots,\lceil\theta\rceil$,

$H_{\theta}^{(j)}(x)=x^{\theta-j}\left(k^{j-\theta} H_{\theta}^{(j)}(k)-\theta \int_{k}^{x} z^{j-\theta-1} H_{\theta}^{(j)}(z-1) \mathrm{d} z\right), \quad k<x \leq k+1(k \in \mathbb{N})$,

thus generalizing the familiar (numerically useful) representation of the $\operatorname{GD}(\theta)$ density function given, e.g. in [1, Equation (4.26)] or [13, Equation (16)].

The formulae for $H_{\theta}^{(j)}(x)$ suggest a Taylor expansion of order $\lceil\theta\rceil-1$ around $a>0$ :

$$
H_{\theta}(x)=\sum_{j=0}^{\lceil\theta\rceil-1} \frac{H_{\theta}^{(j)}(a)}{j !}(x-a)^{j}+\frac{H_{\theta}^{(\lceil\theta\rceil)}(\xi)}{\lceil\theta\rceil !}(x-a)^{\lceil\theta\rceil}, \quad x>0
$$

where $\xi$ is some point between $x$ and $a$. A natural choice would be $a=\theta$ (the mean). An application to the approximation of the GD(5) distribution and density functions is illustrated in Figure 1, indicating an area for further investigation.

\section{Appendix A. Sketch of the proof of Proposition 2.1}

Before we begin, we stress that an analogous but simpler (one-variable version) proof of Proposition 2.1 can be made with respect to the alternative representation of the integrals provided by Remark 2.1. Moreover, cases (i) and (ii) can be handled indirectly but analogously by means of (2.10), thus avoiding the induction step below.

We first need some more notation. Let $\varphi$ be a $C^{0}(\mathbb{R})$ function with $\varphi(0)=0$ and derivative $\varphi^{\prime} \in C^{0}((0, \infty))$, and assume that $\varphi^{\prime}$ and $v$ (a continuous, infinite, Lévy measure) are related according to one of the conditions (i)-(iii) in Proposition 2.1, where $\varphi^{\prime}$ plays the role of $F^{(n)}(\cdot ; t)$. Accordingly, if $\varphi^{\prime}$ is unbounded near $0^{+}$, we shall set $v\left(\mathrm{~d} u_{i}\right)=\rho\left(u_{i}\right) \mathrm{d} u_{i}$ (recall especially the 
sentence after (2.20)). For $k=1,2, \ldots$, we define a two-variable function $W_{k}(\cdot, \cdot)$ on the set

$$
E_{k}=\left\{(x, u): s \leq u \leq \frac{x}{k}\right\}
$$

recursively by

$$
W_{1}(x, u)=\int_{u}^{x} \varphi\left(x-u_{1}\right) v\left(\mathrm{~d} u_{1}\right), \quad(x, u) \in E_{1},
$$

and

$$
W_{k+1}(x, u)=\int_{u}^{x /(k+1)} W_{k}\left(x-u_{1}, u_{1}\right) v\left(\mathrm{~d} u_{1}\right), \quad(x, u) \in E_{k+1} .
$$

Analogously, we define a two-variable function $w_{k}(\cdot, \cdot)$ on $E_{k}$ by replacing the function $\varphi$ with $\varphi^{\prime}$. By definition/convention, $W_{k}(x, x / k)=w_{k}(x, x / k)=0$. With $n$ as in Proposition 2.1 , if we set $\varphi=F^{(j)}(\cdot ; t)$ for some $j=0, \ldots, n-1$ then it holds that $W_{k}(x, s)=$ $A_{s ; k}^{(j)}(x ; t)$ and $w_{k}(x, s)=A_{s ; k}^{(j+1)}(x ; t), x>k s$. We then have the following lemma, from which Proposition 2.1 readily follows.

Lemma A.1. The partial derivative $\partial W_{k} / \partial x$ exists and is equal to $w_{k}$ on $E_{k}$, where $\partial W_{k} / \partial x$ is interpreted as the right partial derivative when $u=x / k$. Moreover, $w_{k}$ is continuous on $E_{k}$.

For clarity and brevity, we omit details that can be worked out easily by the reader.

Sketch of the proof of Lemma A.1. First of all, we note that the case ' $\varphi$ ' is unbounded and absolutely integrable near $0^{+}$' can and will be reduced to the case ' $\varphi^{\prime}$ ' is unbounded and nonnegative near $0^{+}$'. This follows by considering the standard decomposition $\varphi^{\prime}=\left(\varphi^{\prime}\right)^{+}-$ $\left(\varphi^{\prime}\right)^{-}$, which in turn leads to $\varphi=\varphi_{1}-\varphi_{2}$, where $\varphi_{1,2}(x)=\int_{0}^{x}\left(\varphi^{\prime}\right)^{ \pm}(z) \mathrm{d} z$.

Now, the proof is by induction on $k$. We first consider the case in which $k=1$, and let $(x, u)$ be an arbitrary point in $E_{1}$ such that $u<x$. We further assume that $\varphi^{\prime}$ is unbounded near $0^{+}$. The bounded case is much easier to verify. By (A.1) we write $\partial W_{1}(x, u) / \partial x=\lim _{h \rightarrow 0} \hat{w}_{1}(x, u ; h)$, where

$$
\hat{w}_{1}(x, u ; h)=\frac{1}{h}\left(\int_{u}^{x+h} \varphi\left(x+h-u_{1}\right) \rho\left(u_{1}\right) \mathrm{d} u_{1}-\int_{u}^{x} \varphi\left(x-u_{1}\right) \rho\left(u_{1}\right) \mathrm{d} u_{1}\right) .
$$

In what follows we shall consider only the right partial derivative,

$$
\frac{\partial^{+} W_{1}(x, u)}{\partial x}=\lim _{h \rightarrow 0^{+}} \hat{w}_{1}(x, u ; h) .
$$

The left partial derivative is handled the same way. Using the triangle inequality and the mean value theorem applied to $\varphi$, we then have

$$
\begin{aligned}
\left|\hat{w}_{1}(x, u ; h)-w_{1}(x, u)\right| \leq & \left|\int_{u}^{x-\varepsilon}\left(\varphi^{\prime}\left(x-u_{1}+\theta h\right)-\varphi^{\prime}\left(x-u_{1}\right)\right) \rho\left(u_{1}\right) \mathrm{d} u_{1}\right| \\
& +\left|\frac{1}{h} \int_{x-\varepsilon}^{x}\left(\varphi\left(x+h-u_{1}\right)-\varphi\left(x-u_{1}\right)\right) \rho\left(u_{1}\right) \mathrm{d} u_{1}\right| \\
& +\left|\int_{x-\varepsilon}^{x} \varphi^{\prime}\left(x-u_{1}\right) \rho\left(u_{1}\right) \mathrm{d} u_{1}\right| \\
& +\left|\frac{1}{h} \int_{x}^{x+h} \varphi\left(x+h-u_{1}\right) \rho\left(u_{1}\right) \mathrm{d} u_{1}\right|, \quad h>0
\end{aligned}
$$


where $\theta=\theta\left(x-u_{1} ; h\right) \in(0,1)$ and $\varepsilon \in(0, x-u)$. It can be easily shown that the last three terms on the right-hand side of the inequality in (A.3) can be made arbitrary small by choosing first $\varepsilon$, and then $h$, sufficiently small. (For the case corresponding to condition (ii), it is advisable to use the equality $\int_{0}^{\varepsilon}\left(\varphi\left(u_{1}+h\right)-\varphi\left(u_{1}\right)\right) \mathrm{d} u_{1}=\int_{\varepsilon}^{\varepsilon+h} \varphi\left(u_{1}\right) \mathrm{d} u_{1}-\int_{0}^{h} \varphi\left(u_{1}\right) \mathrm{d} u_{1}$; whereas for the case corresponding to (iii), the key idea is to use integration by parts.) Furthermore, for a fixed $\varepsilon$, the first term in this inequality tends to 0 as $h \rightarrow 0^{+}$by uniform continuity of $\varphi^{\prime}$ away from $0^{+}$. Thus, $\partial^{+} W_{1}(x, u) / \partial x=w_{1}(x, u)$. As for the case $u=x$, we just have to consider the last term in (A.3).

The next step is to show that $w_{1}$ is continuous on $E_{1}$. Again we split into the two cases $u<x$ and $u=x$, and proceed according to the definition of continuity. The first case is easily handled using the ' $\varepsilon$-strategy' as above. The second case is straightforward.

So far we have only treated the base case, $k=1$. Now suppose that the lemma holds for some $k \geq 1$ and consider the function $W_{k+1}$ defined on $E_{k+1}$. Without loss of generality, as will turn out below, we can assume the case where $\varphi^{\prime}$ is bounded near $0^{+}$. We first consider the case in which $u<x /(k+1)$. Then, by (A.2), $\partial W_{k+1}(x, u) / \partial x=\lim _{h \rightarrow 0} \hat{w}_{k+1}(x, u ; h)$, where

$$
\begin{gathered}
\hat{w}_{k+1}(x, u ; h)=\frac{1}{h}\left(\int_{u}^{(x+h) /(k+1)} W_{k}\left(x+h-u_{1}, u_{1}\right) v\left(\mathrm{~d} u_{1}\right)\right. \\
\left.-\int_{u}^{x /(k+1)} W_{k}\left(x-u_{1}, u_{1}\right) v\left(\mathrm{~d} u_{1}\right)\right) .
\end{gathered}
$$

Here, as before, we shall consider only the right partial derivative. The left partial derivative is handled similarly. Writing down $w_{k+1}(x, u)$ according to relation (A.2), and then using the triangle inequality and the mean value theorem when applied to $W_{k}$ in the first coordinate (recalling the induction hypothesis), we obtain

$$
\begin{aligned}
\left|\hat{w}_{k+1}(x, u ; h)-w_{k+1}(x, u)\right| \leq & \left|\int_{u}^{x /(k+1)}\left(w_{k}\left(x-u_{1}+\theta h, u_{1}\right)-w_{k}\left(x-u_{1}, u_{1}\right)\right) v\left(\mathrm{~d} u_{1}\right)\right| \\
& +\left|\frac{1}{h} \int_{x /(k+1)}^{(x+h) /(k+1)} W_{k}\left(x+h-u_{1}, u_{1}\right) v\left(\mathrm{~d} u_{1}\right)\right|, \quad h>0
\end{aligned}
$$

where $\theta=\theta\left(u_{1} ; x ; h\right) \in(0,1)$. Applying the second part of the induction hypothesis, $w_{k}$ is uniformly continuous on bounded subsets of $E_{k}$. Hence, the first term on the right-hand side of the inequality in (A.4) tends to 0 as $h \rightarrow 0^{+}$. Noting that $W_{k}\left(k u_{1}, u_{1}\right)=0$ and applying the mean value theorem, the same also holds for the second term. We conclude that $\partial^{+} W_{k+1}(x, u) / \partial x=w_{k+1}(x, u)$. As for the case $u=x /(k+1)$, we just have to consider the second term.

As the final step of the sketched proof, we need to show that $w_{k+1}$ is continuous on $E_{k+1}$. Again we split into the two cases $u<x /(k+1)$ and $u=x /(k+1)$, and proceed according to the definition of continuity after applying relation (A.2) to $w_{k+1}$. Both cases follow straightforwardly under the uniform continuity of $w_{k}$ mentioned above.

\section{Appendix B. A generalization of Theorem 2.1 and Corollary 2.1}

Although Theorem B.1 and Corollary B.1, below, are much stronger than their counterparts, Theorem 2.1 and Corollary 2.1, we preferred to put them in an appendix, primarily since the 
generalization was discovered after the paper was ready for acceptance. Moreover, the original approach has its own merits and the extension to the arbitrary subordinator measure $\pi$ has no added value in the context of the present paper.

Theorem B.1. Let $\mu^{t}$ and $\mu_{s}^{t}$ denote the distributions at time $t>0$ of a pure-jump subordinator with Lévy measure $\pi$ and $\pi_{\mid(0, s]}$ respectively, and set $\Lambda(s)=\pi((s, \infty))$ and $\lambda_{s}(\mathrm{~d} y)=$ $\mathbf{1}_{(s, \infty)}(y) \pi(\mathrm{d} y)$. Then $\mu_{s}^{t}$ is given by

$$
\mu_{s}^{t}=\mathrm{e}^{t \Lambda(s)} \sum_{k=0}^{\infty} \frac{(-t)^{k}}{k !}\left(\mu^{t} * \lambda_{s}^{* k}\right)
$$

$\left(\lambda_{s}^{* 0}\right.$ is the Dirac measure at 0$)$. In particular, the distribution function $\mu_{s}^{t}([0, x])$ is given, for $x \geq 0$, by

$$
\mu_{s}^{t}([0, x])=\mathrm{e}^{t \Lambda(s)}\left(\mu^{t}([0, x])+\sum_{k=1}^{\lceil x / s\rceil-1} \frac{(-t)^{k}}{k !} \int_{(k s, x]} \mu^{t}([0, x-u]) \lambda_{s}^{* k}(\mathrm{~d} u)\right)
$$

Alternatively, with $D_{s ; k}(x)$ defined as before,

$$
\begin{aligned}
\mu_{s}^{t}([0, x])=\mathrm{e}^{t \Lambda(s)} & \left(\mu^{t}([0, x])\right. \\
& \left.+\sum_{k=1}^{\lceil x / s\rceil-1} \frac{(-t)^{k}}{k !} \int_{D_{s ; k}(x)} \mu^{t}\left(\left[0, x-\sum_{i=1}^{k} u_{i}\right]\right) \pi\left(\mathrm{d} u_{1}\right) \cdots \pi\left(\mathrm{d} u_{k}\right)\right) .
\end{aligned}
$$

Proof. Let us denote by $L_{\sigma}$ the Laplace transform of a measure $\sigma$ on $[0, \infty)$, i.e. $L_{\sigma}(\omega)=$ $\int_{[0, \infty)} \mathrm{e}^{-\omega x} \sigma(\mathrm{d} x), \omega \geq 0$. Substituting the Laplace transforms of the distributions $\mu_{s}^{t}$ and $\mu^{t}$ according to (1.1), we have

$$
\begin{aligned}
L_{\mu_{s}^{t}}(\omega) & =L_{\mu^{t}}(\omega) \exp \left[-t \int_{(s, \infty)}\left(\mathrm{e}^{-\omega x}-1\right) \pi(\mathrm{d} x)\right] \\
& =L_{\mu^{t}}(\omega) \mathrm{e}^{t \Lambda(s)} \exp \left[-t L_{\lambda_{s}}(\omega)\right] \\
& =\mathrm{e}^{t \Lambda(s)}\left(\sum_{k=0,2, \ldots} \frac{t^{k}}{k !} L_{\mu^{t}}(\omega)\left[L_{\lambda_{s}}(\omega)\right]^{k}-\sum_{k=1,3, \ldots} \frac{t^{k}}{k !} L_{\mu^{t}}(\omega)\left[L_{\lambda_{s}}(\omega)\right]^{k}\right) \\
& =L_{\sigma_{1}}(\omega)-L_{\sigma_{2}}(\omega),
\end{aligned}
$$

where $\sigma_{1}$ and $\sigma_{2}$ are finite measures on $[0, \infty)$ defined by

$$
\sigma_{1}=\mathrm{e}^{t \Lambda(s)} \sum_{k=0,2, \ldots} \frac{t^{k}}{k !}\left(\mu^{t} * \lambda_{s}^{* k}\right), \quad \sigma_{2}=\mathrm{e}^{t \Lambda(s)} \sum_{k=1,3, \ldots} \frac{t^{k}}{k !}\left(\mu^{t} * \lambda_{s}^{* k}\right) .
$$

Thus, $L_{\mu_{s}^{t}+\sigma_{2}}(\omega)=L_{\sigma_{1}}(\omega)$ and in turn, by uniqueness of Laplace transforms, $\mu_{s}^{t}+\sigma_{2}=\sigma_{1}$; that is, $\mu_{s}^{t}=\sigma_{1}-\sigma_{2}$ and (B.1) is proved. Equation (B.2) follows immediately, and in turn we obtain its alternative form, (B.3), by virtue of (2.10). 
Corollary B.1. Suppose that $\mu^{t}$, and, hence, $\mu_{s}^{t}$, is absolutely continuous. Let $\rho^{t}$ and $\rho_{s}^{t}$ denote the corresponding densities. Then $\rho_{s}^{t}$ is given, for $x>0$, by

$$
\begin{aligned}
\rho_{s}^{t}(x) & =\mathrm{e}^{t \Lambda(s)}\left(\rho^{t}(x)+\sum_{k=1}^{\lceil x / s\rceil-1} \frac{(-t)^{k}}{k !} \int_{(k s, x]} \rho^{t}(x-u) \lambda_{s}^{* k}(\mathrm{~d} u)\right) \\
& =\mathrm{e}^{t \Lambda(s)}\left(\rho^{t}(x)+\sum_{k=1}^{\lceil x / s\rceil-1} \frac{(-t)^{k}}{k !} \int_{D_{s ; k}(x)} \rho^{t}\left(x-\sum_{i=1}^{k} u_{i}\right) \pi\left(\mathrm{d} u_{1}\right) \cdots \pi\left(\mathrm{d} u_{k}\right)\right) .
\end{aligned}
$$

Proof. As in the proof of Corollary 2.1, the proof follows from

$$
\int_{(k s, x]} \int_{u}^{x} \rho^{t}(z-u) \mathrm{d} z \lambda_{s}^{* k}(\mathrm{~d} u)=\int_{k s}^{x} \int_{(k s, z]} \rho^{t}(z-u) \lambda_{s}^{* k}(\mathrm{~d} u) \mathrm{d} z
$$

(verified by Tonelli's theorem).

\section{Acknowledgements}

I am very grateful to Mr and Mrs Shapack for funding my fellowship, and to my supervisor Professor Ely Merzbach for all his help and advice. I am much indebted to the anonymous referee for a thorough reading of the manuscript, for many constructive comments that led to a substantial improvement in its presentation, and for several valuable mathematical observations.

\section{References}

[1] Arratia, R., Barbour, A. D. and Tavaré, S. (2003). Logarithmic Combinatorial Structures: A Probabilistic Approach. European Mathematical Society, Zürich.

[2] Asmussen, S. And Rosiński, J. (2001). Approximations of small jumps of Lévy processes with a view towards simulation. J. Appl. Prob. 38, 482-493.

[3] Basawa, I. V. And Brockwell, P. J. (1982). Nonparametric estimation for nondecreasing Lévy processes. J. R. Statist. Soc. B 44, 262-269.

[4] Covo, S. (2008). On approximations of small jumps of subordinators with particular emphasis on a Dickman-type limit. Submitted.

[5] Eliazar, I. And Klafter, J. (2003). On the extreme flights of one-sided Lévy processes. Physica A 330, 8-17.

[6] Emmer, S. And Klüppelberg, C. (2004). Optimal portfolios when stock prices follow an exponential Lévy process. Finance Stoch. 8, 17-44.

[7] Griffiths, R. C. (1988). On the distribution of points in a Poisson Dirichlet process. J. Appl. Prob. 25, 336-345.

[8] Hensley, D. (1986). The convolution powers of the Dickman function. J. London Math. Soc. 33, 395-406.

[9] Holst, L. (2001). The Poisson-Dirichlet distribution and its relatives revisited. Preprint, Royal Institute of Technology, Stockholm. Available at http://www.math.kth.se/matstat/.

[10] Howlader, H. A. and Balasooriya, U. (2003). Bayesian estimation of the distribution function of the Poisson model. Biometrical J. 45, 901-911.

[11] Jacod, J. And Shiryaev, A. N. (2003). Limit Theorems for Stochastic Processes, 2nd edn. Springer, Berlin.

[12] Madan, D. B., CARr, P. P. And Chang, E. C. (1998). The variance gamma process and option pricing. Europ. Finance Rev. 2, 79-105.

[13] Penrose, M. D. and Wade, A. R. (2004). Random minimal directed spanning trees and Dickman-type distributions. Adv. Appl. Prob. 36, 691-714.

[14] Perman, M. (1993). Order statistics for jumps of normalised subordinators. Stoch. Process. Appl. 46, $267-281$.

[15] Pitman, J. and Yor, M. (1997). The two-parameter Poisson-Dirichlet distribution derived from a stable subordinator. Ann. Prob. 25, 855-900.

[16] Rosiński, J. (2001). Series representations of Lévy Processes from the perspective of point processes. In Lévy Processes, eds O. E. Barndorff-Nielsen et al. Birkhäuser, Boston, MA, pp. 401-415.

[17] Sato, K.-I. (1999). Lévy Processes and Infinitely Divisible Distributions (Camb. Stud. Adv. Math. 68). Cambridge University Press. 
[18] TAKács, L. (1955). On stochastic processes connected with certain physical recording apparatuses. Acta Math. Acad. Sci. Hungar. 6, 363-380.

[19] VervaAt, W. (1972). Success Epochs in Bernoulli Trials (with Applications in Number Theory). Mathematisch Centrum, Amsterdam.

[20] WheELer, F. S. (1990). Two differential-difference equations arising in number theory. Trans. Amer. Math. Soc. 318, 491-523. 